\title{
Venus Express: Highlights of the Nominal Mission ${ }^{1}$
}

\author{
D. V. Titov ${ }^{a, b}$, H. Svedhem ${ }^{c}$, F. W. Taylor ${ }^{d}$, S. Barabash ${ }^{e}$ J.-L. Bertaux ${ }^{f}$, P. Drossart ${ }^{g}$, \\ V. Formisano ${ }^{h}$, B. Häusler ${ }^{i}$, O. Korablev ${ }^{b}$, W. J. Markiewicz ${ }^{a}$, D. Nevejans ${ }^{j}$, M. Pätzold ${ }^{k}$, \\ G. Piccionil, J.-A. Sauvaud ${ }^{m}$, T. L. Zhang ${ }^{n}$, O. Witasse ${ }^{c}$, J.-C. Gerard ${ }^{o}$, A. Fedorov \\ A. Sanchez-Lavega ${ }^{q}$, J. Helbert ${ }^{r}$, and R. Hoofs ${ }^{s}$ \\ ${ }^{a}$ Max-Planck Institute for Solar System Research, Katlenburg-Lindau, Germany \\ ${ }^{b}$ Space Research Institute (IKI), Moscow, Russia \\ ${ }^{c}$ ESA/ESTEC, Noordwijk, The Netherlands \\ ${ }^{d}$ Oxford University, $U K$ \\ ${ }^{e}$ IRF, Kiruna, Sweden \\ ${ }^{f}$ Service d'Aeronomie, CNRS, France \\ ${ }^{g}$ LESIA, Observatoire de Paris, Meudon, France \\ ${ }^{h}$ IFSI/INAF, Rome, Italy \\ ${ }^{i}$ Universitute der Bundeswehr, München, Germany \\ ${ }^{j}$ Institute for Space Aeronomy, Belgium \\ ${ }^{k}$ Rheinean Institute for Environmental research, Universitute zu Küln, Cologne, Germany \\ ${ }^{l}$ IASF/CNR, Rome, Italy \\ ${ }^{m}$ Centre d'Etude Spatiale des Rayonnements, Tolouse, France \\ ${ }^{n}$ Space Research Institute, Graz, Austria \\ ${ }^{\circ}$ LPAP, Universite de Liege, Belgium \\ ${ }^{p}$ CESR, Toulouse, France \\ ${ }^{q}$ Universidad del Pais Vasco, Bilbao, Spain \\ ${ }^{r}$ Institute for Planetary Research, DLR, Berlin, Germany \\ ${ }^{s}$ ESA/ESAC, Villanueva de la Canada, Madrid, Spain \\ e-mail: titov@mps.mpg.de
}

Received October 7, 2008

\begin{abstract}
Venus Express is the first European (ESA) mission to the planet Venus. Its main science goal is to carry out a global survey of the atmosphere, the plasma environment, and the surface of Venus from orbit. The payload consists of seven experiments. It includes a powerful suite of remote sensing imagers and spectrometers, instruments for in-situ investigation of the circumplanetary plasma and magnetic field, and a radio science experiment. The spacecraft, based on the Mars Express bus modified for the conditions at Venus, provides a versatile platform for nadir and limb observations as well as solar, stellar, and radio occultations. In April 2006 Venus Express was inserted in an elliptical polar orbit around Venus, with a pericentre height of $\sim 250 \mathrm{~km}$ and apocentre distance of $\sim 66000 \mathrm{~km}$ and an orbital period of 24 hours. The nominal mission lasted from June 4 , 2006 till October 2, 2007, which corresponds to about two Venus sidereal days. Here we present an overview of the main results of the nominal mission, based on a set of papers recently published in Nature, Icarus, Planetary and Space Science, and Geophysical Research Letters.
\end{abstract}

PACS numbers: 96.30.Ea, 96.12.Jt, 96.12.Kz, 96.12.Ma, 96.12.Wx

DOI: $10.1134 / \mathrm{S} 0038094609030010$

\section{INTRODUCTION}

Despite the fact that about 25 spacecraft visited Venus in the 20th century, a great number of fundamental problems in its physics remained unsolved (Moroz, 2002; Titov et al., 2006a; Taylor et al., 2006). This was already recognized by the end of the 1990's, however,

\footnotetext{
${ }^{1}$ The article is published in the original.
}

none of the world space agencies planned another mission to the planet, leading to that Venus was nicknamed: the "forgotten planet". After more than a decade, the European Space Agency (ESA) was the first to respond to this challenge by initiating Venus Express-the first European mission to Venus. The mission aims at a global remote and in-situ investigation of the atmosphere, plasma environment, and some surface properties from 
orbit. In particular, fundamental problems in the fields of atmospheric structure and composition, morphology and distribution of clouds and hazes, atmospheric dynamics, properties of plasma and magnetic field, and escape processes, are all addressed by the Venus Express observations. These investigations will shed light on the current climate of Venus, will make it possible to reconstruct the evolutionary path of our sister planet, and will make significant contribution to the field of comparative planetology.

The Venus Express payload consists to a great extent of the instruments inherited from the Mars Express and Rosetta missions, which were found to be quite suitable for making a breakthrough in Venus studies (Titov et al., 2001). The payload core consists of a suite of imagers and spectrometers, combining wide spectral range from the UV to the thermal IR. Broad band context imaging and high spectral resolution makes the mission payload the most capable remote sensing package ever flown to Venus. This suite is complemented by a radio science experiment to investigate the structure of the neutral atmosphere and ionosphere. Two experiments monitor the ambient magnetic field and study the plasma environment and escape processes.

The mission re-uses the Mars Express bus modified for the conditions at Venus (Svedhem et al., 2007a). The spacecraft provides a versatile 3 -axis stabilized platform to carry out various observations. These include nadir observations along almost the entire orbit, tracking the planetary limb and selected spots on the surface, as well as solar, stellar and Earth radio occultations that require high precision and stability of pointing. The broad spectrum of mission goals, complexity of the payload, and operational limitations due to re-use of the Mars Express spacecraft that had been originally designed for the colder environment at Mars, justified the need for careful planning of observations in order to effectively use the spacecraft and payload capabilities and to maximize overall science return (Titov et al., 2006c). Coordination of the observations between different experiments onboard the spacecraft is a particular feature of Venus Express science planning. This synergistic approach makes it possible to effectively address the science objectives of the mission using several experiments.

The nominal Venus Express mission lasted from June 4, 2006 till October 2, 2007, which corresponds to about two Venus sidereal days. This paper gives an overview of the results recently published in a special section of Nature, in several papers in Geophysical Research Letters, and in Icarus. The results are grouped in topics to demonstrate payload synergy in tackling certain scientific problems. We also outline future investigations that are already being carried out or planned for the extended mission (October 3, 2007December 31, 2009).

\section{SPACECRAFT, PAYLOAD, ORBIT, MISSION SCENARIO AND OBSERVATIONS}

The Venus Express spacecraft design is based on the successful Mars Express bus-a $600 \mathrm{~kg} 3$-axis stabilized platform with two body-fixed telecommunication antennas (Fig. 1). The spacecraft is described in detail by Svedhem et al. (2007a, 2008). Most of the subsystems and payloads are mounted on its walls and on panels inside the bus, while ASPERA and MAG are accommodated outside the spacecraft. The propulsion system is located on the bottom of the bus on the $-Z$ side. The $+X$ wall is almost completely occupied by the $1.3 \mathrm{~m}$ dish of the main high-gain antenna (HGA1). The opposite $(-X)$ side of the bus carries payload cryogenic radiators. The $Y$ walls carry the solar panels and radiators that cool the equipment and some of the instruments. The small $0.3 \mathrm{~m}$ dish of the second high-gain antenna (HGA2) and the MAG boom are mounted on the top $+Z$ floor. The apertures of the optical instruments are also located on this wall. The spacecraft can withstand only limited solar illumination on all walls except for $+Z$ and $+X$, which greatly adds to the complexity of planning the observations.

The Venus Express payload consists of seven instruments (Fig. 1, table), including three spectrometers (SPICAV/SOIR, PFS, and VIRTIS) operating in broad spectral range from UV to thermal $1 R$ to study the structure, composition, and dynamics of the atmosphere in nadir geometry. For the first time, spectral imaging in the near-IR range routinely provides an orbital survey of the composition of the lower atmosphere, the structure of the deep cloud, and the surface, through night-side imaging in the spectral transparency "windows" by VIRTIS and VMC. Also for the first time, SPICAV/SOIR uses stellar and solar occultation technique at Venus for precise investigation of the structure and composition of the mesosphere and upper haze. These observations are complemented by the limb imaging by VIRTIS and VMC. Venus Monitoring Camera takes wide angle context images in four channels to study morphology and dynamics at the cloud tops. Both VIRTIS and VMC provide thermal mapping of the surface. The ASPERA-4 experiment investigates in-situ plasma and energetic neutral atoms (ENA) around the planet to characterize escape processes. It is supported by MAG measurements of the magnetic field. The standard radio system of the spacecraft, complemented by an ultra-stable oscillator (USO), constitutes the radio science experiment (VeRa) that studies the fine structure of the atmosphere and ionosphere in Earth radio occultation mode, investigates the surface with bi-static radar and gravity anomaly experiments, and sounds the solar corona. Unfortunately, due to a failure in the scanning mechanism, the Planetary Fourier Spectrometer (PFS) is currently not operational. The programme of observations was adjusted so that the other instruments could partially compensate for this loss.

SOLAR SYSTEM RESEARCH Vol. 43 No. 32009 


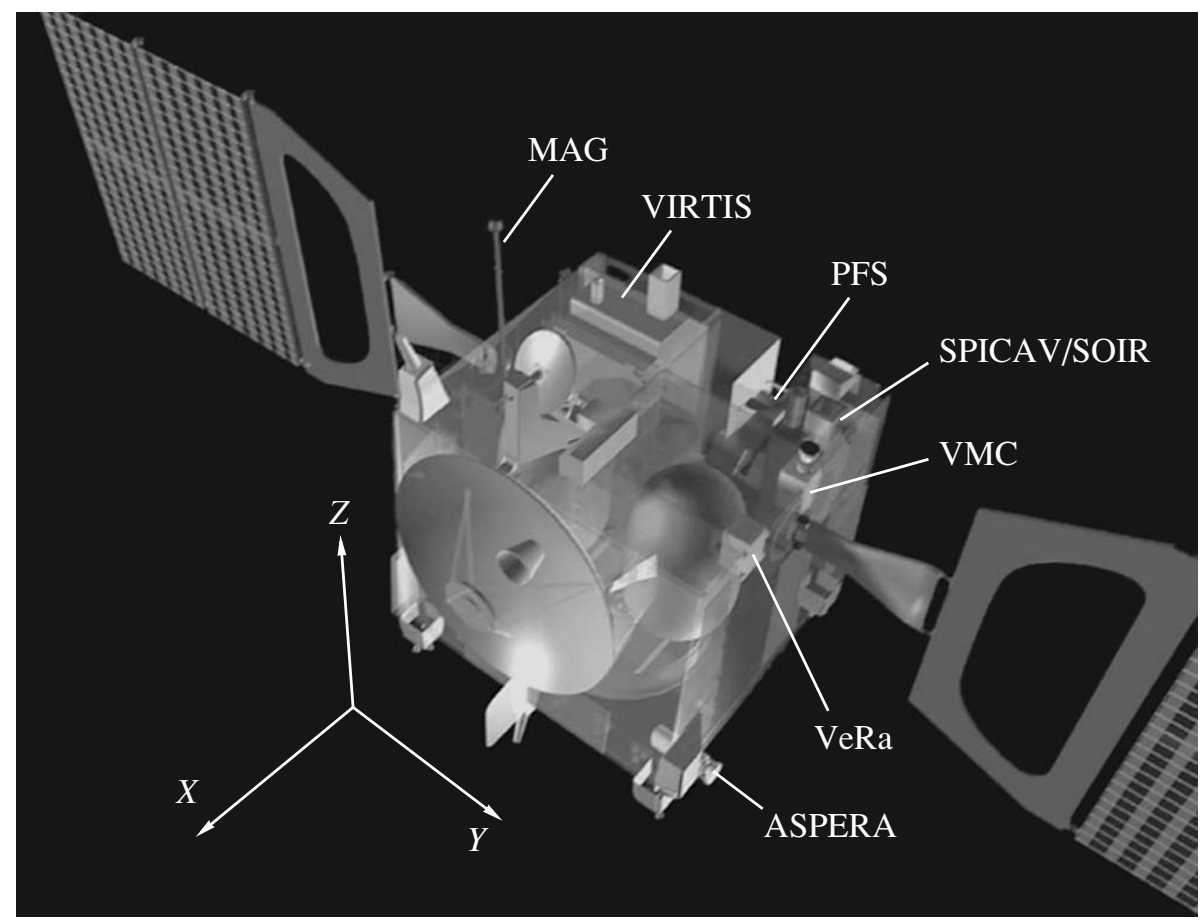

Fig. 1. Semi-transparent view of the Venus Express spacecraft with science instruments shown.

The optical axes of the instruments are aligned with the $+Z$ axis of the spacecraft (Fig. 1). Thus, the main observation mode is the one with the $+Z$ axis looking at the planet. Solar occultation requires the SPICAV solar port, which points 30 degrees off the $+Y$ direction, to look at the Sun. In the radio-occultation experiment the big high-gain antenna aligned with the $+X$ axis points to the Earth. The dense atmosphere bends the path of the microwave rays considerably, and special spacecraft attitude steering is required to compensate for this and to ensure correct pointing during a complete occultation, which can last up to 50 minutes. In the bi-static sounding experiment the antenna points at a selected surface target and the reflected waves are recorded by

Scientific payload of Venus Express

\begin{tabular}{|c|c|c|c|}
\hline Name (acronym) & Description & Measured parameters & References \\
\hline ASPERA-4 & $\begin{array}{l}\text { In-situ detection and characterization } \\
\text { of neutral and charged particles }\end{array}$ & $\begin{array}{l}\text { Electrons } 0.01-15 \mathrm{keV} \text {; ions } 0.01- \\
36 \mathrm{keV} / \mathrm{q} \text {; neutral particles } 0.1-60 \mathrm{keV} \\
\text { and } 0.1-10 \mathrm{keV}\end{array}$ & $\begin{array}{l}\text { Barabash et al., 2007a } \\
\text { Barabash et al., } 2008\end{array}$ \\
\hline MAG & $\begin{array}{l}\text { Dual sensor fluxgate magnetometer, } \\
\text { one sensor on } 1 \mathrm{~m} \text { boom }\end{array}$ & Magnetic field $8 \mathrm{pT}-262 \mathrm{nT}$ at $128 \mathrm{~Hz}$ & $\begin{array}{l}\text { Zhang et al., } 2006 \\
\text { Zhang et al., } 2008\end{array}$ \\
\hline PFS & $\begin{array}{l}\text { Planetary Fourier Spectrometer (not } \\
\text { operating) }\end{array}$ & $\begin{array}{l}\text { Wavelengths } 0.9-45 \mu \mathrm{m} \text {, spectral resolu- } \\
\text { tion } \sim 1 \mathrm{~cm}^{-1}\end{array}$ & $\begin{array}{l}\text { Formisano et al., } 2006 \\
\text { Formisano et al., } 2008\end{array}$ \\
\hline SPICAV/SOIR & $\begin{array}{l}\text { Ultraviolet and near infrared spec- } \\
\text { trometer for stellar and solar occulta- } \\
\text { tion and nadir observations }\end{array}$ & $\begin{array}{l}\text { Wavelengths } 110-320 \mathrm{~nm}, 0.7-1.65 \mu \mathrm{m} \\
\text { and } 2.2-4.4 \mu \mathrm{m} \text {; spectral resolving power } \\
\text { up to } 20000\end{array}$ & $\begin{array}{l}\text { Bertaux et al., } 2007 \mathrm{a} \\
\text { Bertaux et al., } 2008\end{array}$ \\
\hline VeRa & Radio science investigation & $\begin{array}{l}\mathrm{X} \text { - and S-band Doppler shift, polarization } \\
\text { and amplitude variations }\end{array}$ & $\begin{array}{l}\text { Häusler et al., } 2006 \\
\text { Häusler et al., } 2008\end{array}$ \\
\hline VIRTIS & $\begin{array}{l}\text { Ultraviolet-visible-infrared imaging } \\
\text { and high-resolution infrared spec- } \\
\text { trometer }\end{array}$ & $\begin{array}{l}\text { Wavelength } 0.25-5 \mu \mathrm{m} \text { for the imaging } \\
\text { spectrometer and } 2-5 \mu \mathrm{m} \text { for the high-res- } \\
\text { olution channel; resolving power about } \\
2000\end{array}$ & $\begin{array}{l}\text { Drossart et al., 2007a } \\
\text { Piccioni et al., } 2008\end{array}$ \\
\hline VMC & $\begin{array}{l}\text { Venus Monitoring Camera for wide- } \\
\text { angle imaging }\end{array}$ & $\begin{array}{l}\text { Four parallel narrow band channels at } \\
365,513,965 \text {, and } 1010 \mathrm{~nm}\end{array}$ & $\begin{array}{l}\text { Markiewicz et al., 2007a } \\
\text { Markiewicz et al., } 2008\end{array}$ \\
\hline
\end{tabular}




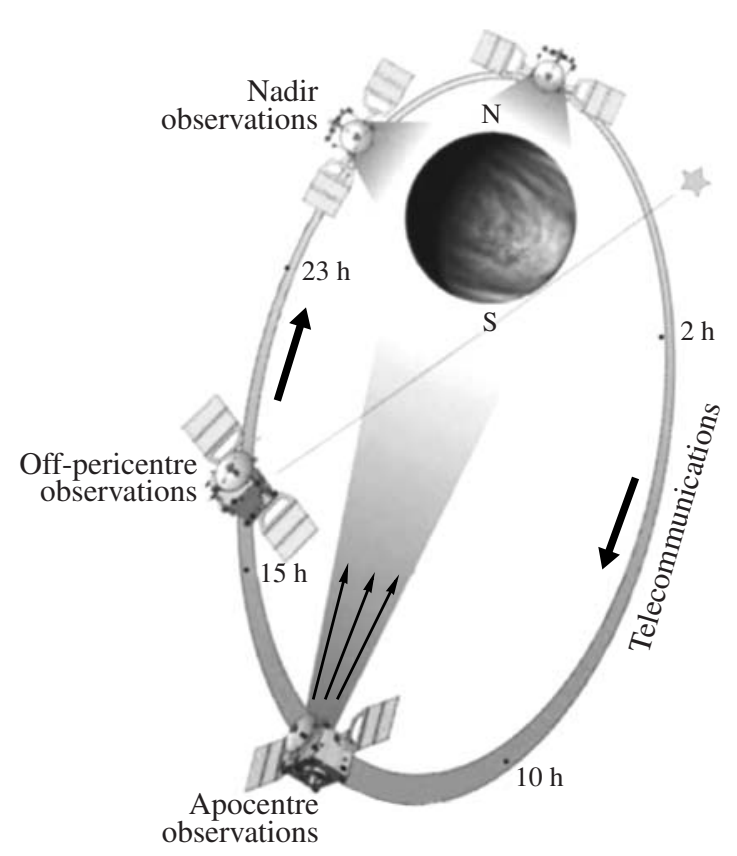

Fig. 2. Sketch of the Venus Express orbit. Figures along the orbit show the time since pericentre passage (orbital time).

the ground station. Precise steering manoeuvres will also be conducted in this case, to provide specular reflection for the selected targets.

Venus Express was launched on November 9, 2005 from the Baykonur cosmodrome in Kazakhstan by the Russian Soyuz-Fregat launcher. On April 11, 2006 the spacecraft was successfully inserted into a highly elliptical polar orbit around Venus with pericentre at $\sim 78^{\circ}$ N. Several corrective manoeuvres brought Venus Express to the final orbit with a period of 24 hours and an apocentre distance of $\sim 66000 \mathrm{~km}$ (Fig. 2). This orbit, firstly, allows observations of atmospheric phenomena at different local times, and, secondly, combines a global view of the planet in the Southern hemisphere with high-resolution snapshots in the North. The pericentre slowly drifts towards the Northern pole with a rate of $\sim 4$ degrees per year. In addition, the gravity of the Sun perturbs the orbit, forcing the pericentre altitude to increase with a rate of $1-2 \mathrm{~km}$ per day. Specific manoeuvres are regularly performed to maintain the pericentre altitude in the $250-350 \mathrm{~km}$ range.

The Venus Express orbit can be roughly divided in three parts (Fig. 2): pericentre observations (23-2 hour orbital time), telecommunications on the descending arc of the orbit (2-12 hours), and apocentre and off-pericentre observations in the ascending arc (12-23 hours). The phase of the orbit is maintained so that the ground station in Cebreros, Spain is visible from the satellite between 2 and 12 hours orbital time.

After about 50 days of spacecraft and payload commissioning, the mission entered its nominal phase that lasted for about 500 Earth days (2 Venus sidereal days) from June 4, 2006 till October 2, 2007. Figure 3 shows the nominal mission overview in Sun-Earth fixed coordinates.

Observations usually start at the apocentre, and continue along the ascending arc of the orbit and during the pericentre pass (Fig. 2). Thus in each orbit the observations cover almost all latitudes in nadir geometry. Local time at the ascending node of the orbit, which approximately corresponds to 23 hours orbital time in Fig. 2, slowly changes from orbit to orbit. Since the nominal mission lasted for two Venus sidereal days, all local times on tte planet were observed twice. About $30 \mathrm{UV}$ bright stars were used by SPICAV for occultation studies of the upper atmosphere. Since the satellite orbit is fixed relative to motionless stars, each star sounds certain latetude all along the mission at different local time. The total number of stellar occultations performed during the nominal mission exceeds 100 . They cover latitude range from $50^{\circ} \mathrm{S}$ to $40^{\circ} \mathrm{N}$.

Solar and Earth occultations are grouped in seasons, each of them lasting for $\sim 50$ days (Fig. 3). During these periods, occultation soundings have priority and are scheduled in at least every second orbit, each having both ingress and egress observations, one in the Southern and the other in the Northern hemisphere. Five eclipse seasons and three Earth occultation seasons occurred in the nominal mission (Fig. 3). During this first stage Venus Express carried out 98 solar and 116 Earth radio occultations. Figures 4 shows the latitudelocal solar time coverage for Earth occultations. Solar occultations occur at the terminator, thus sounding morning and evening sectors of the planet. Figure 5 shows the coverage in solar occultations during the nominal mission.

Although the payload and the spacecraft are in good shape in general, and observations continue to be made, several problems have resulted in degradation of the science return. The first was that the PFS scanning mechanism got stuck and the instrument, although perfectly functional, cannot see the planet. While the PFS science goals related to observations in the near IR range were partially recovered by VIRTIS and SPICAV, the thermal part of the Venus spectrum between 5 and $45 \mu \mathrm{m}$ was lost. This had a negative impact on mesospheric temperature sounding and on measurements of the structure and composition of the cloud tops. These goals have been partially recovered by VIRTIS thermal sounding in the $4.3 \mu \mathrm{m} \mathrm{CO}$ band and more intensive use of the radio-science experiment VeRa. The second problem that occurred near the end of the nominal mission was the loss of the S-band signal in the telecommunication system. For this reason the bi-static sounding of the surface and solar corona investigation has not been possible since summer 2007. Radio occultation measurements of the neutral atmosphere and ionosphere were not affected by this loss, although the interpretation of dispersive atmospheric absorption properties became more cumbersome. The third problem was 


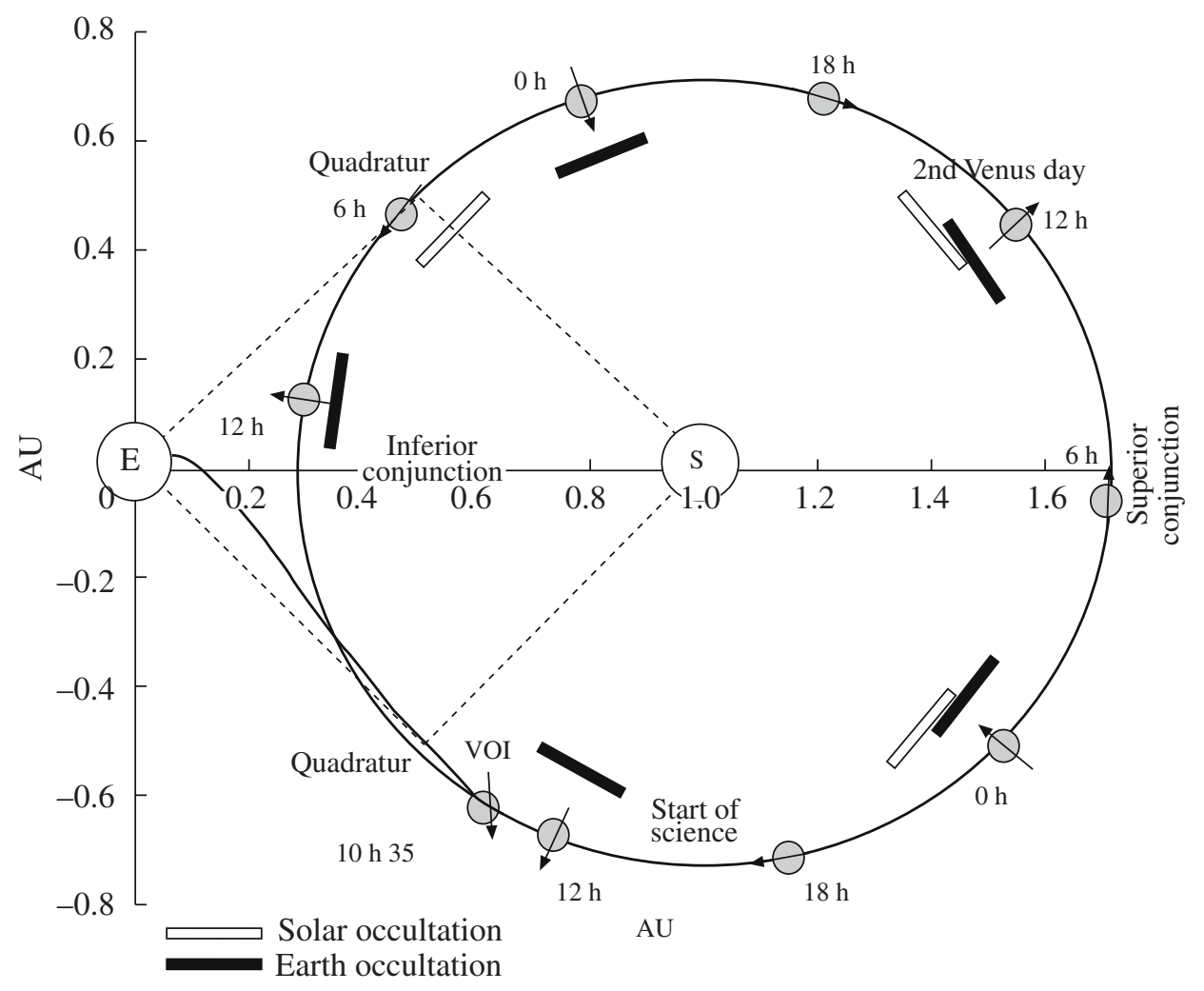

Fig. 3. Overview of the nominal mission. Sun and Earth are fixed and shown with "S" and "E", while Venus moves along the solid line. The arrow crossing the Venus disk shows the direction of the satellite orbital motion. Bars along the Venus orbit mark solar (solid bars) and Earth (open bars) occultation seasons, VOI means "Venus Orbit Insertion".

damage to the VMC detector by direct solar illumination during the approach to Venus in March-April 2006. This resulted in the appearance of a complex and evolving sensitivity pattern on the CCD detector. A reasonably good correction was achieved by additional flat fielding using images taken in the same orbit close to pericentre.

\section{STRUCTURE OF THE ATMOSPHERE}

Three methods of remote temperature sounding are used by Venus Express. Earth radio occultation provides temperature structure of the mesosphere and upper troposphere down to the cloud bottom $(90-40 \mathrm{~km})$ with vertical resolution of few hundred metres (Häusler et al., 2006). Latitude and local solar time coverage achieved in this experiment during the nominal mission is shown in Fig. 4. The mesosphere $(90-60 \mathrm{~km})$ is also studied by VIRTIS thermal emission spectroscopy in the $4.3 \mu \mathrm{m} \mathrm{CO}_{2}$ band (Drossart et al., 2007a). This method gives broader spatial coverage but vertical resolution is limited to 2-3 kilometres. Also accurate temperature determination by VIRTIS has been possible so far for the night side only. Application of this technique on the day side requires accurate modelling of reflected solar radiation, since this cannot be neglected at these wavelengths. The least studied region, the upper mesos- phere-lower thermosphere (140-90 km), is sounded for the first time in stellar occultation geometry by SPICAV (Bertaux et al., 2007a). Together these three techniques cover the altitude range from $140 \mathrm{~km}$ down to 40 $\mathrm{km}$.

Figure 6 shows examples of temperature structure measured by the Earth radio occultation (Pätzold et al., 2007) and stellar occultations (Bertaux et al., 2007b) techniques, revealing unprecedented detail. The most remarkable are the strong latitude variability of the temperature structure above the clouds, and the existence of a quasi-isothermal layer whose thickness increases from few kilometres at low latitudes to almost $15 \mathrm{~km}$ in polar regions. The temperature increases with a constant lapse rate of $\sim 10 \mathrm{~K} / \mathrm{km}$ below the tropopause $(\sim 60 \mathrm{~km})$.

SPICAV discovered a warm layer at the mesopause $(\sim 100 \mathrm{~km})$ (Fig. 6b), in which the amplitude of the temperature peak increases from the terminator region towards midnight. This behaviour was interpreted as a result of adiabatic heating in the downwelling branch of the solar-antisolar thermospheric circulation on the night side.

Figure 7 shows the altitude-latitude temperature fields from the VIRTIS thermal-IR sounding. Two prominent features of the temperature field are obvious. Temperature at the same altitude level increases from 


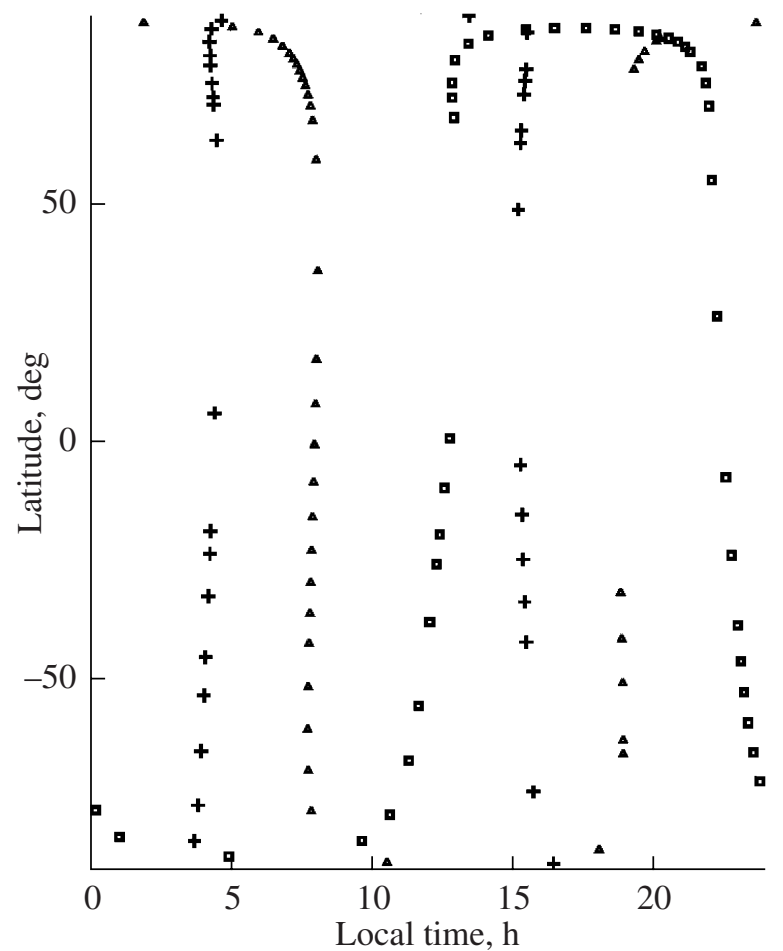

Fig. 4. Latitude-local solar time coverage in the Earth radio occultations during the nominal mission. Symbols mark three occultation seasons: no. 1 (2006)-pluses, no. 2 (2006-2007)-triangles, no. 3 (2007)-squares.

equator to pole, implying radiative non-equilibrium and suggesting a significant role of atmospheric dynamics in maintaining such temperature structure. The second feature is the existence of a region with strong (up to $20 \mathrm{~K}$ ) temperature inversions at $\sim 70 \mathrm{~km}$ in latitude range from $50^{\circ}$ to $70^{\circ} \mathrm{S}$. The discovery of these two peculiarities of the temperature field in the Southern hemisphere, similar to those found in the Northern hemisphere by earlier missions (Taylor et al., Lellouch et al., 1997; Zasova et al., 2007), indicates global hemispheric symmetry of the Venus temperature field.

Figure 6 compares the Venus Express observations to the VIRA model (Sieff et al., 1985), and shows that the observations deviate from the model predictions. In particular, this relates to the temperatures in the thermosphere, where SPICAV found much higher temperatures with strong diurnal variations. The VIRA model needs to be updated to incorporate the Venus Express results.

\section{ATMOSPHERIC COMPOSITION}

Venus Express uses two novel techniques to study the atmospheric composition. SPICAV/SOIR acquires high-resolution $(\lambda / \Delta \lambda \sim 20000)$ spectra of the Venus atmosphere in solar occultation geometry (Bertaux et al., 2007a), providing vertical profiles of atmospheric trace gases in the mesosphere $(110-70 \mathrm{~km})$. This region features vigorous chemistry and dynamics that were

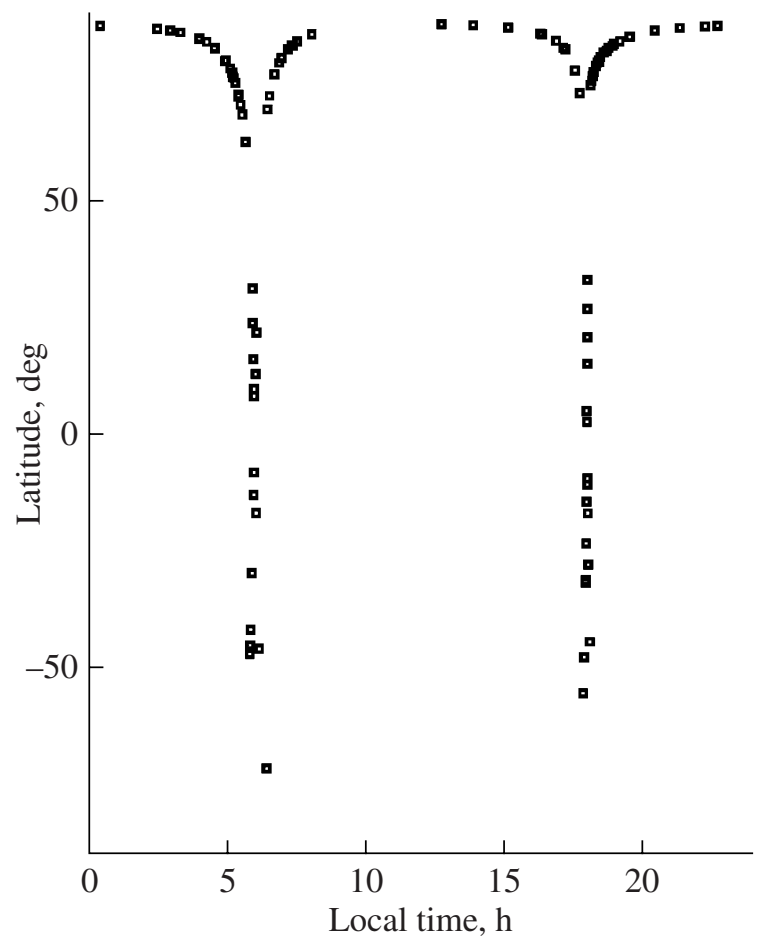

Fig. 5. Latitude — local solar time coverage in solar occultations by SOIR/SPICAV.

poorly studied in the earlier missions. Spectral signatures of $\mathrm{CO}_{2}, \mathrm{CO}, \mathrm{H}_{2} \mathrm{O}, \mathrm{HDO}, \mathrm{HF}, \mathrm{HCl}, \mathrm{SO}_{2}$ are clearly identified in the SOIR spectra (Bertaux et al., 2007b). Figure 8 shows examples of $\mathrm{H}_{2} \mathrm{O}$ and $\mathrm{HDO}$ vertical profiles derived from the SOIR occultation sounding.

Venus Express exploits the potential of deep atmosphere sounding through the spectral transparency "windows" on the night side discovered by Allen and Crawford (1984). VIRTIS maps the Venus dark side with moderate spectral resolution at $0.9-5.0 \mu \mathrm{m}$ and simultaneously takes high-resolution spectra between 2 and $4 \mu \mathrm{m}$ (Drossart et al., 2007a). These observations provide a global survey of the lower atmosphere composition, monitoring the abundance of $\mathrm{CO}, \mathrm{COS}, \mathrm{SO}_{2}$, $\mathrm{H}_{2} \mathrm{O}$ at $30-40 \mathrm{~km}, \mathrm{HCl}$ and $\mathrm{H}_{2} \mathrm{O}$ at $\sim 23 \mathrm{~km}$ and $\mathrm{H}_{2} \mathrm{O}$ close to the surface. Interpretation of these spectra requires accurate radiative transfer modelling in the deep atmosphere of Venus. Here we present a preliminary analysis of these measurements. Figure 9 shows an overview of the Venus Express composition findings (Svedhem et al., 2007b). Vertical profiles of the minor species above the clouds are derived from the SPICAV/SOIR solar occultation sounding. The abundance of $\mathrm{CO}, \mathrm{COS}, \mathrm{H}_{2} \mathrm{O}, \mathrm{SO}_{2}$ below the clouds are obtained from the VIRTIS near-IR spectra on the night side.

Venus Express composition measurements have several important implications. Knowledge of the vertical profiles of the trace gases is important in order to 


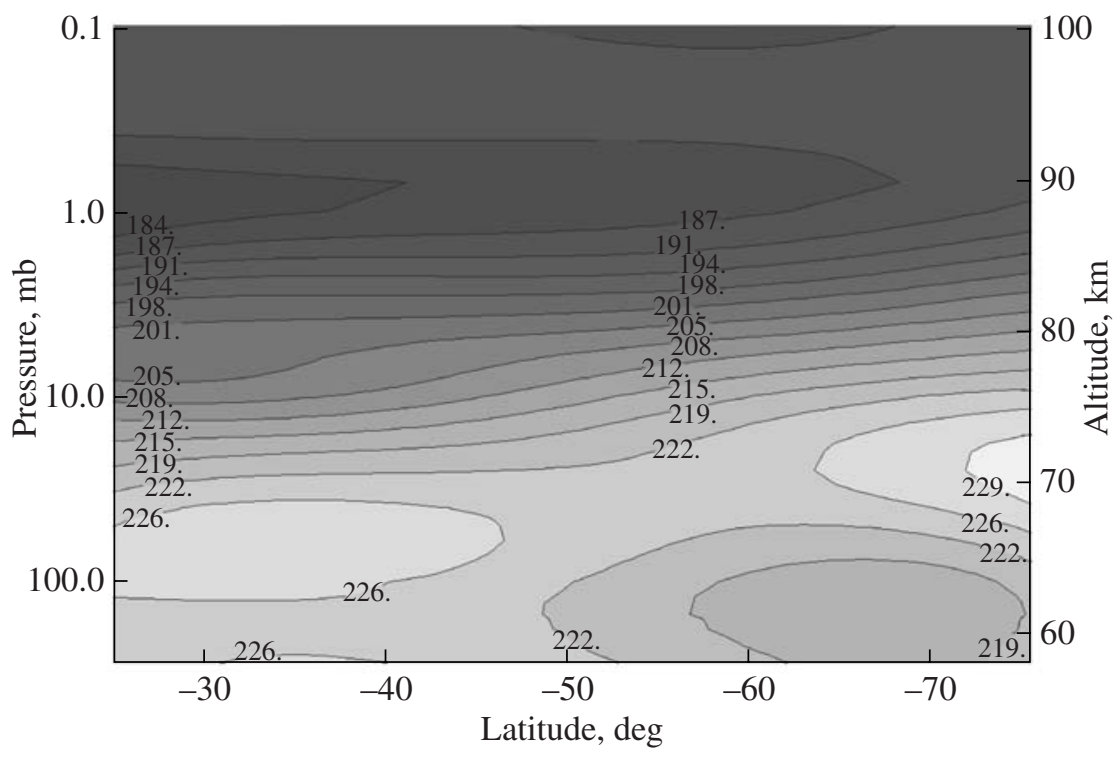

Fig. 7. Temperature field in the Southern hemisphere derived from VIRTIS thermal emission spectroscopy. The retrievals are reliable above $\sim 65 \mathrm{~km}$. (D. Grassi, personal communication).

constrain models of mesospheric chemistry and photochemistry. In addition, the abundance of hydrogenbearing species is relevant to the ultimate escape of $\mathrm{H}$ and evolution of the atmosphere. The SOIR measurements give $\mathrm{HF}$ abundance that are lower by a factor of 3 , and HCI mixing ratios that are 4-6 times lower, compared to the earlier observations. One possible explanation of low level of these chemically active species is that the global circulation can bring air depleted in these gases from higher altitudes. This should occur in the descending branch of the Hadley cell at high latitudes, where SPICAV carried out most of its solar occultation sounding.

The $\mathrm{D} / \mathrm{H}$ ratio is an important tracer of the escape processes and has particular relevance for the history of water on Venus. Earlier observations yielded a strong enhancement $(\mathrm{D} / \mathrm{H} \sim 150)$ of heavy water molecule in the troposphere of Venus as compared to Earth (Donahue and Russell, 1997). The simultaneous observations of both $\mathrm{H}_{2} \mathrm{O}$ and $\mathrm{HDO}$ by SOIR/Venus Express give vertical profile of $\mathrm{D} / \mathrm{H}$ ratio in the mesosphere right below the altitudes from which escape occurs. At $\sim 70 \mathrm{~km}$ the measured ratio is close to that in the bulk atmosphere and tends to increase with altitude by a factor of $\sim 2.5$, which could be a sign of preferential escape of $\mathrm{H}$ atoms when deuterium is left behind in excess to recombine with $\mathrm{OH}$ radicals. If this interpretation is correct, the SOIR measurements are the first indication of differential escape of $\mathrm{H}$ versus $\mathrm{D}$ acting at present, a process that could explain the high $\mathrm{D} / \mathrm{H}$ ratio in the present atmosphere of Venus. The observed presence of HDO in the photo-dissociation region also indicates that $\mathrm{D}$ atoms must be present in the thermosphere, where they would be expected to experience non-thermal escape along with $\mathrm{H}$ atoms.

The Venus Express observations have confirmed earlier, controversial detections of the existence of lightning on Venus. Russell et al. (2007) reported the

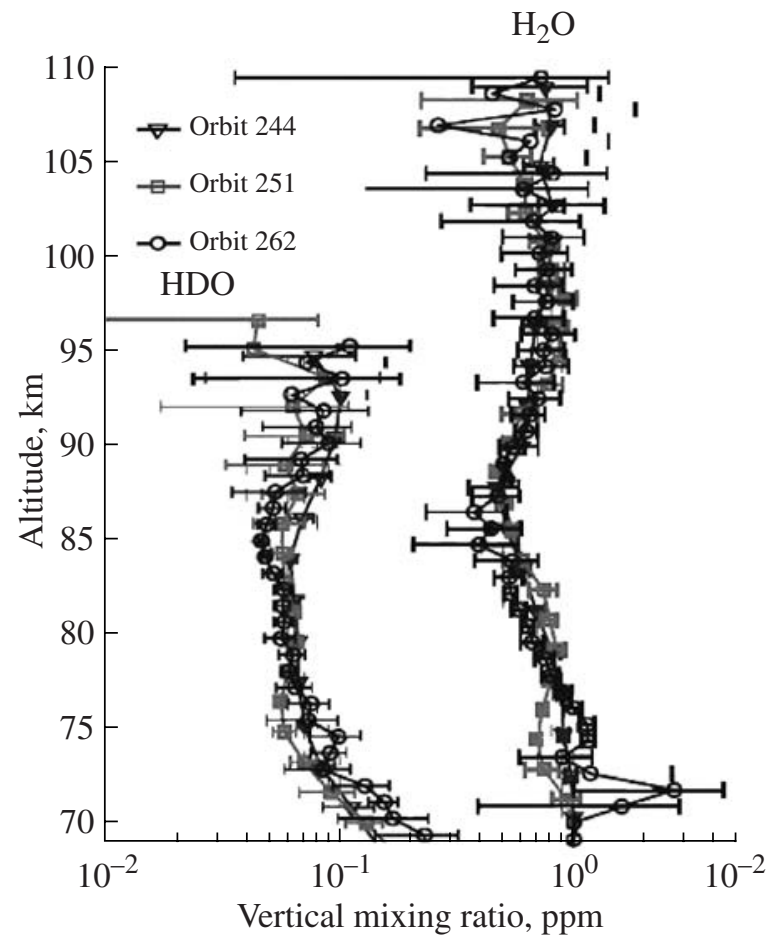

Fig. 8. Vertical profiles of $\mathrm{H}_{2} \mathrm{O}$ and $\mathrm{HDO}$ mixing ratios derived from SPICAV/SOIR sounding in three orbits. 


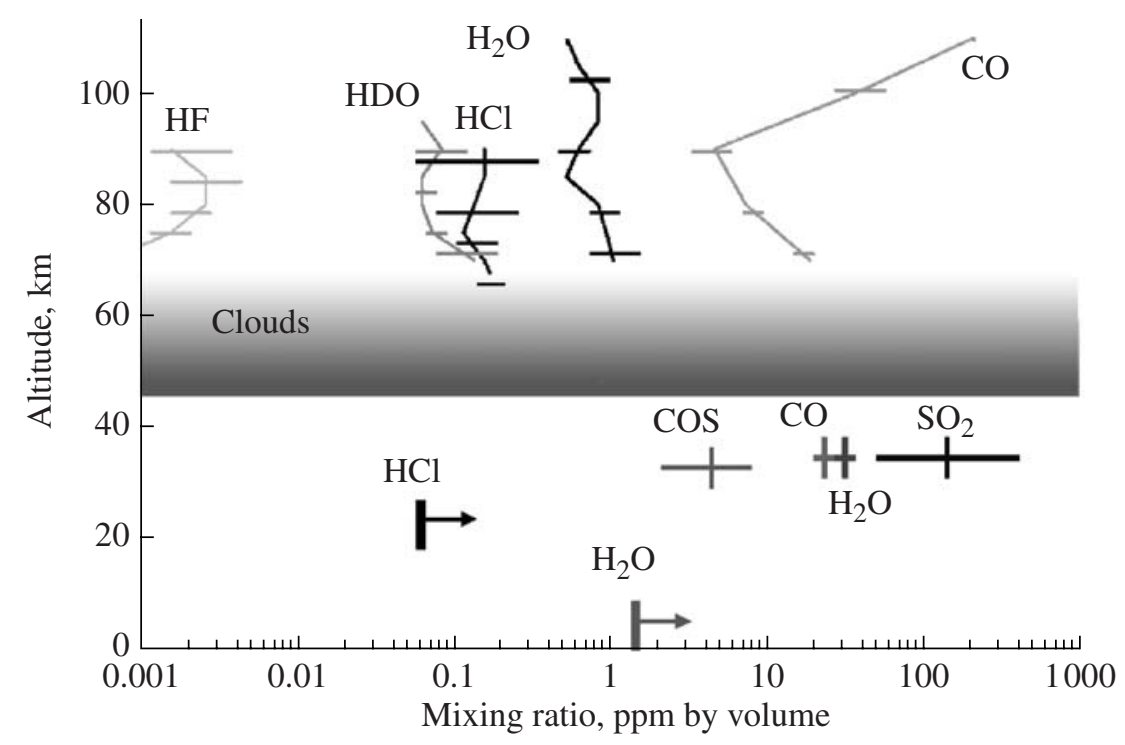

Fig. 9. Atmospheric composition from the Venus Express observations. Error bars in mixing ratios give the range of maximum and minimum detections over all latitudes, and those in altitude indicate the width of weighting functions. The bars with arrows show expected sensitivity of the Venus Express measurements for which data analysis is still in progress.

(A)

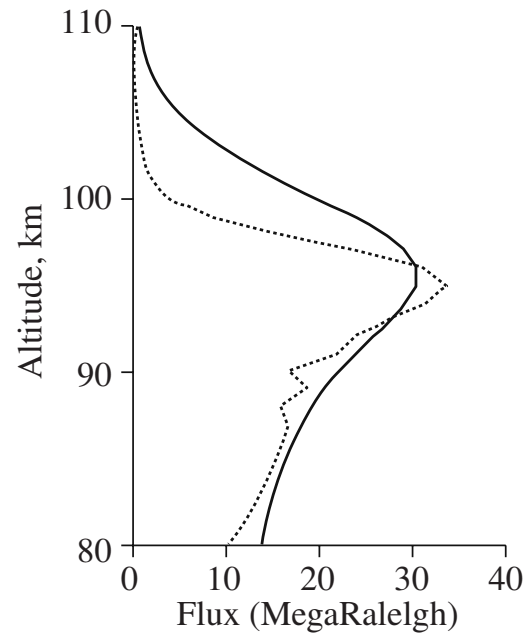

(B)

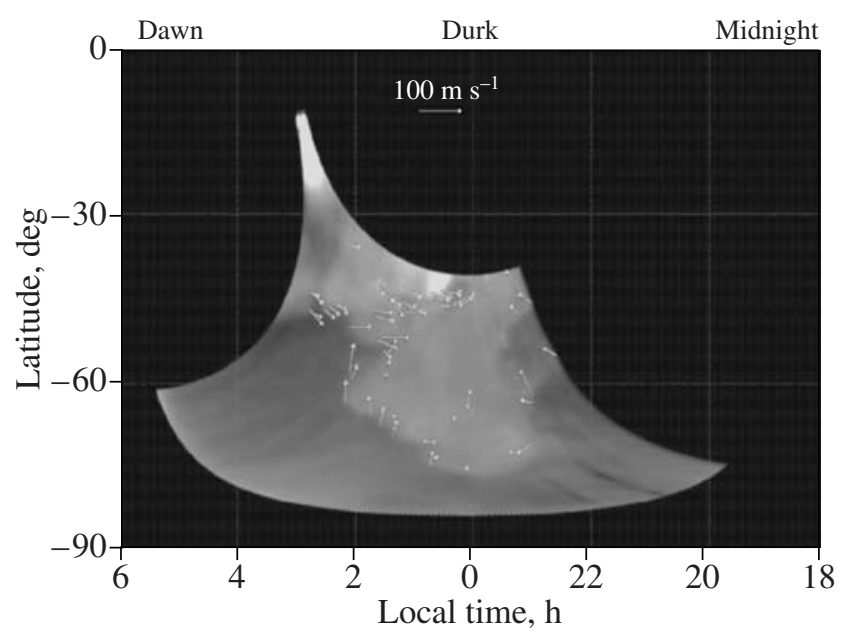

Fig. 10. (A) The limb profile of the $\mathrm{O}_{2}\left({ }^{1} \Delta_{\mathrm{g}}\right)$ emission flux observed by VIRTIS in orbit no. 76 at $35^{\circ} \mathrm{N}$ (orange) compared to the predictions of a one-dimensional photochemical-diffusive transport model (blue). (B) Map of the $\mathrm{O}_{2}$ emission captured by VIRTIS in orbit no. 84 in nadir geometry. Vectors show apparent motions of the emission features.

definitive detection of electromagnetic, whistler-mode waves propagating from the atmosphere to the ionosphere. The magnetometer observations revealed strong, circularly polarized, electromagnetic waves with frequencies near $100 \mathrm{~Hz}$. The waves appeared as bursts of radiation lasting 0.25 to $0.5 \mathrm{~s}$, and have the expected properties of whistler-mode signals generated by lightning discharges in Venus' clouds. The estimated mean global frequency was found to be close to that on the Earth. The occurrence of lightning in a planetary atmosphere enables chemical processes to take place that would not occur under standard temperatures and pressures, and that in turn can lead to the formation of "disequilibrium" chemical species.

\section{NON-LTE EMISSIONS}

Several non-LTE emissions from Venus were discovered by the earlier ground-based and space-borne observations. The most important are the NO emissions in UV (Feldman et al., 1979; Stewart et al., 1980), the $\mathrm{O}_{2}$ near-infrared airglow (Connes et al., 1979; Crisp 
(a)

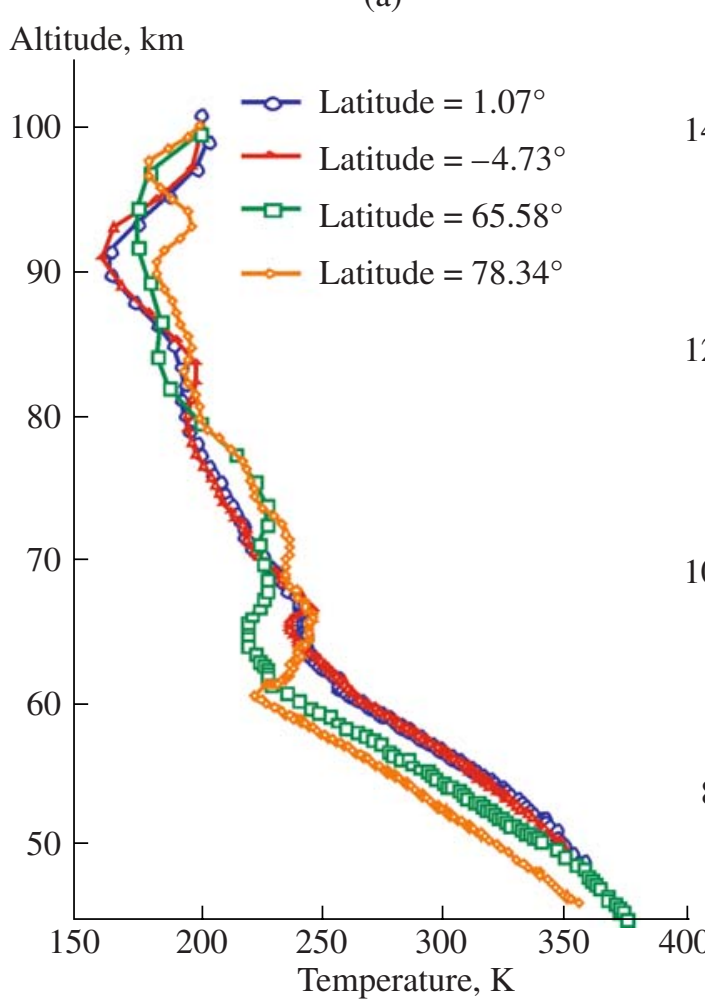

Altitude, km

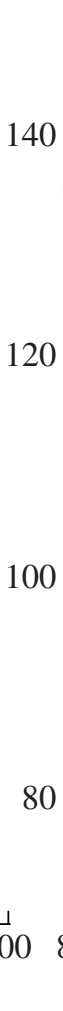

240 -

220 (b)

--- Ref.7

口 Ploneer Venus Night Probe 1978

- Ref.6

- Orbit $95\left(\mathrm{SZA}=121^{\circ}\right)$

- Orbit $96\left(\right.$ SZA $\left.=122^{\circ}\right)$

- Orbit $98\left(\mathrm{SZA}=124^{\circ}\right)$

- Orbit $102\left(\mathrm{SZA}=167^{\circ}\right)$

-Orbit $103\left(\mathrm{SZA}=167^{\circ}\right)$

-Orbit $104\left(\mathrm{SZA}=170^{\circ}\right)$

.n.m. Radio Occ Magelan

$\left(\mathrm{SZA}=109^{\circ}\right)$

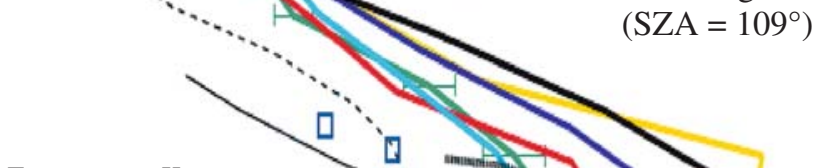

Temperature, $\mathrm{K}$

$200-\quad 8$

0

0

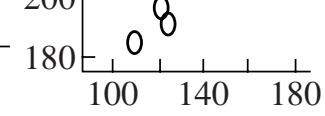

5

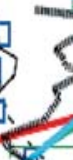

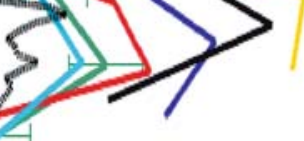

in B shows the dependence of mesospheric peak temperature on the solar zenith angle (SZA).

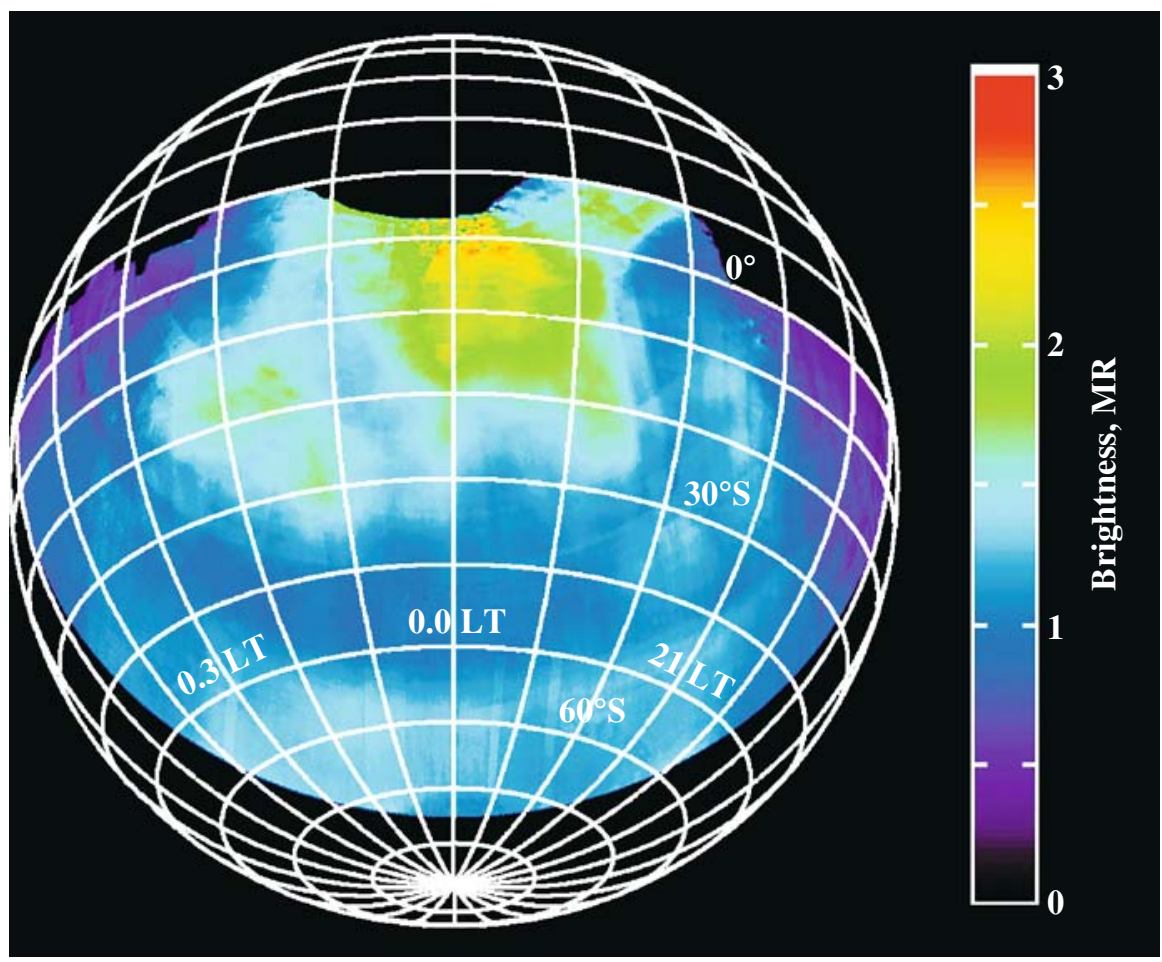

Fig. 11. Global map of the vertical brightness (in MR) of the $\mathrm{O}_{2}$ infrared nightglow observed by VIRTIS at low solar activity. The emission rate accounts for the component reflected by the clouds and the emission angle. The perspective is from $30^{\circ}$ in the midnight meridian. Parallels are indicated every $10^{\circ}$ and local time meridians are separated by 1 hour. 
et al., 1996; Ohtsuki, S. et al., 2005), and dayside emissions from $\mathrm{CO}_{2}$ and $\mathrm{CO}$ (Lopez-Valverde et al., 2007). The emissions originate in the upper atmosphere (90$140 \mathrm{~km}$ ) from the processes of radiative recombination and fluorescence. At these altitudes the pressure, and hence the collision rate, is low, so that molecules in excited energy states have enough time to emit a photon before collisions bring them into thermal equilibrium. Observations of these emissions can be used to study conditions and dynamics in the lower mesosphere, but the earlier data lacked temporal and spatial coverage and vertical resolution. The advanced spectro-imaging payload suite, combination of nadir and limb geometry, and highly eccentric polar orbit of Venus Express allow the emissions, their spatial and vertical distribution and variations to be investigated in unprecedented detail.

The $\mathrm{O}_{2}$ near-infrared airglow is produced by threebody recombination of oxygen atoms formed on the day side by photo-dissociation of $\mathrm{CO}_{2}$ and $\mathrm{CO}$. Global circulation transports the oxygen atoms to the night side, where they recombine giving $\sim 75 \%$ of $\mathrm{O}_{2}$ molecules in the ${ }^{1} \Delta_{\mathrm{g}}$ excited state that emits at $\lambda \sim 1.27 \mu \mathrm{m}$. Figure 10 shows the vertical and spatial distribution of the $\mathrm{O}_{2}\left({ }^{1} \Delta_{\mathrm{g}}\right)$ emission observed by VIRTIS (Drossart et al., 2007b). In limb geometry the observations integrate the $\mathrm{O}_{2}$ emission along the line of sight. The altitude of its peak is usually located near $95 \mathrm{~km}$ and is controlled by competition between vertical transport and recombination. The brightness is proportional to the downward flux of oxygen atoms, which was estimated to be $5 \times 10^{11} \mathrm{~cm}^{-2} \mathrm{~s}^{-1}$ for the profile shown in Fig. 10a. The derived maximum atomic oxygen number density, reached at $100 \mathrm{~km}$, is $1.3 \times 10^{11} \mathrm{~cm}^{-3}$. This quantity has not been measured previously by other techniques.

In the nadir mode VIRTIS maps the spatial distribution of the $\mathrm{O}_{2}$ night airglow. Figure 11 shows a global map in which the distribution of the $\mathrm{O}_{2}\left({ }^{1} \Delta_{\mathrm{g}}\right)$ nightglow (Gerard et al., 2008) exhibits significant spatial and temporal variability ranging over an order of magnitude, the brightest emission reaching $\sim 3$ mega-Rayleighs (MR) at low latitudes near the midnight meridian. The nadir brightness averaged over the night side of the Southern hemisphere is $1.3 \mathrm{MR}$, in very good agreement with earlier ground based observations. The Venus Express spacecraft observes non-LTE and airglow phenomena from apocentre above the South Pole, an unique perspective that reveals the night-side emission over most of the southern hemisphere and yields its latitudinal distribution, especially around local midnight. The emissions are observed during solar minimum for the first time, thus complementing the earlier observations.

VIRTIS also observes non-LTE emissions in the $4.3 \mu \mathrm{m} \mathrm{CO}_{2}$ and $4.6 \mu \mathrm{mCO}$ bands on the day side. These emissions originate from the processes of resonance scattering of solar radiation and fluorescence. The emission at $4.3 \mu \mathrm{m} \mathrm{CO}$ band has its maximum intensity located at $90-120 \mathrm{~km}$, depending mainly on

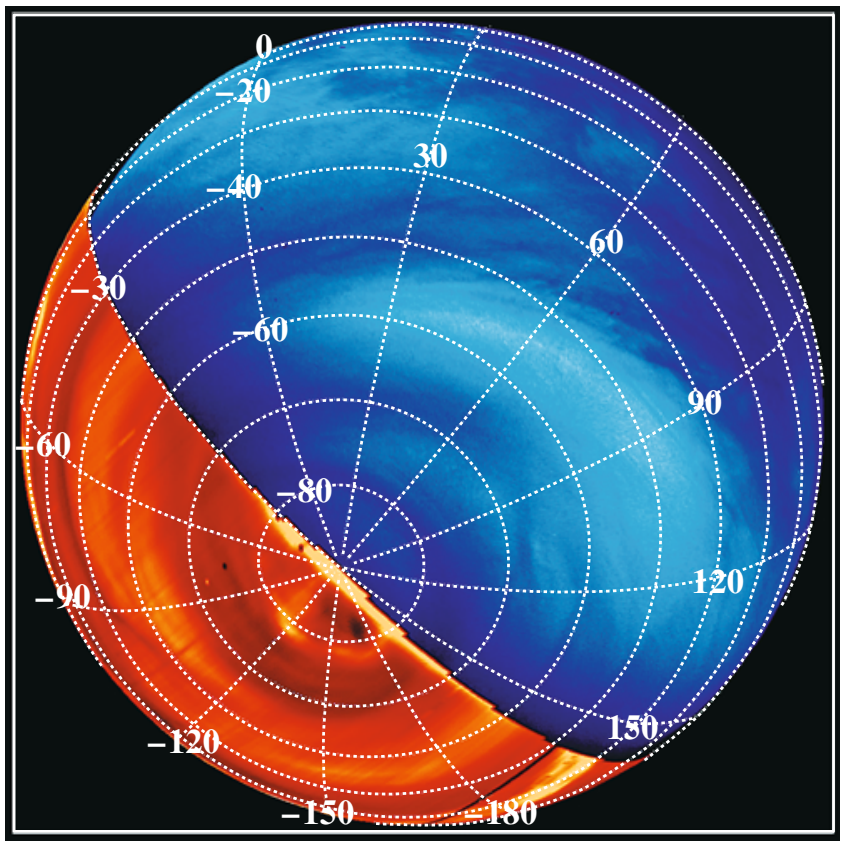

Fig. 12. False colour image of the Venus Southern hemisphere composed of a VMC UV image on the day side (blue) and a VIRTIS near-IR image in the $2.3 \mu \mathrm{m}$ spectral transparency "window" on the night side (red).

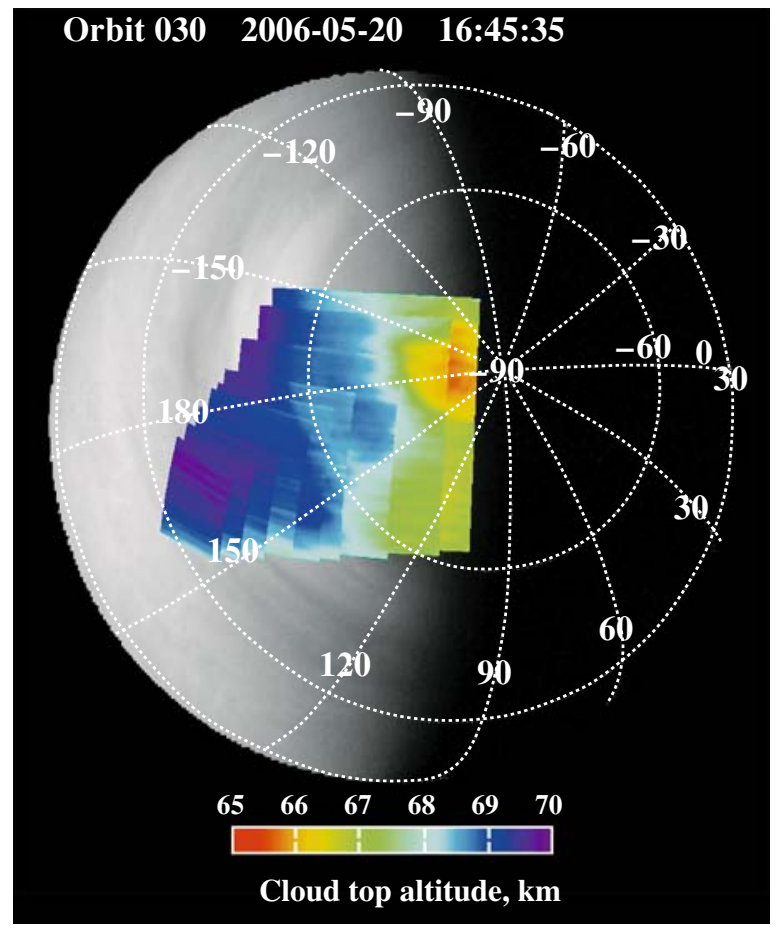

Fig. 15. Altimetry of the cloud tops. The colour mosaic of the cloud top altitude derived from VIRTIS spectral imaging in the near-IR $\mathrm{CO}_{2}$ bands is overplotted on a simultaneously captured VMC UV image (Titov et al., 2008). 
the solar zenith angle. The peak altitude is defined by a competition between the exponentially increasing density of the atmosphere with depth and the increasing collision frequency, which brings the molecules into LTE, quenching this emission. The altitude and the peak brightness are therefore related to the density structure, and their observed variations tell us about atmospheric processes at these altitudes.

\section{CLOUDS AND HAZES}

The spectro-imaging instruments in the Venus Express payload use the highly eccentric polar orbit of the satellite to investigate the Venus cloud layer in the spectral range from UV to thermal IR in unprecedented detail, at all latitudes and local solar times, with spatial resolution ranging from $\sim 50 \mathrm{~km}$ at the apocentre to few hundred metres at pericentre (Svedhem et al., 2007a; Titov et al., 2006b). Multispectral imaging provides for the first time an opportunity to reconstruct the global cloud morphology in 3-D. Also limb observations and stellar/solar occultation techniques allow the instruments to reveal the vertical structure of the upper haze.

Figure 12 shows a synthetic view of the planet produced by combining the VMC UV image on the day side (Markiewicz et al., 2007b) with the VIRTIS image taken in the $2.3 \mu \mathrm{m}$ spectral transparency "window" on the night side (Piccioni et al., 2007). The UV markings are produced by inhomogeneous spatial and vertical distribution of an unknown absorber in the upper cloud, their morphology indicating variations of dynamic state at about $70 \mathrm{~km}$ altitude. At low latitudes $\left(<40^{\circ} \mathrm{S}\right)$ the mottled and patchy cloud pattern suggests the significant role of convection in the vicinity of the equator, where a large fraction of the solar energy is deposited in the upper cloud $(65-55 \mathrm{~km})$ (Titov et al., 2007). Further towards the pole, mottled clouds give way to streaky features, indicating a transition to more regular quasi-laminar flow in the mid-latitudes. The mid-latitudes $\left(50-70^{\circ} \mathrm{S}\right)$ are dominated by a bright, almost featureless, band, implying the presence of a large amount of conservative scattering aerosol that masks the UV absorber. Circular and spiral dark features a few hundred kilometres across appear in the polar regions (Fig. 12). The brightness in the middle and high latitudes shows remarkable variability on a daily time scale. This indicates vigorous dynamical and microphysical processes like nucleation and coagulation that change the upper cloud opacity (Markiewicz et al., 2007b).

The near-IR part of the image in Fig. 12 shows emission leaking from the hot lower atmosphere $(\sim 35 \mathrm{~km})$ through the spectral transparency window at $2.3 \mu \mathrm{m}$ on the night side. Here the contrasts mark spatial variations of the opacity of the main cloud deck at roughly $50-55 \mathrm{~km}$ altitude. Brightness variations by about a factor of 10 roughly correspond to opacity variations between 20 and 40. The opacity contrast is even higher in the $1.7 \mu \mathrm{m}$ "window".
The cloud morphology revealed by Venus Express imaging (Fig. 12) apparently has a global vortex-like organization. Day-side UV and night-side near-IR observations imply that this pattern covers the whole Southern hemisphere, and persists at least down to the cloud bottom $(\sim 50 \mathrm{~km})$. Comparison to earlier observations in the North (Limaye, 2007) suggests remarkable overall hemispheric symmetry of the global cloud pattern on Venus. Moreover, the planetary vortex on Venus has some striking morphological similarities to the hurricanes on Earth (Limaye et al., 2007), although the scales and driving forces are quite different in these two cases.

The highly eccentric orbit of Venus Express allows the imaging instruments to zoom in certain regions and phenomena and to put high resolution images in a global context. Figure 13 shows examples of meso- and small-scale cloud morphology seen in the VMC UV and VIRTIS near-IR images.

Figures 13a, 13b show the details of the transition from patchy clouds at low latitudes to streaky features in middle latitudes which occurs between $40^{\circ}$ and $50^{\circ} \mathrm{S}$. Fine structure of the convective clouds at low latitudes close to the sub-solar point is shown in Fig. 13c. The imaging instruments observed a variety of waves. In the polar regions VMC detected long straight regular features that seemed to induce short waves nearby (Fig. 13d). The waves associated with the spiral arms of the global vortex were seen by VIRTIS in the deep cloud (Fig. 13e).

The most spectacular cloud feature discovered at thermal IR wavelength by VIRTIS is the eye of the planetary vortex in polar latitudes (Fig. 14) (Piccioni et al., 2007). The shape of the feature varies from oval to "S" shaped. The eye is about $1500 \mathrm{~km}$ across and resides within $\sim 70$ degrees circle, rotating around the South pole with a period of $\sim 2.5$ days. Brightness temperature in the eye is $30-40 \mathrm{~K}$ higher than that of its surroundings, from which it is separated by very sharp boundaries. Fine filaments are also clearly visible inside the vortex eye.

The appearance and morphology of the eye suggests that this is a dynamical "singularity" at the centre of the global hemispheric vortex. Infrared sounding revealed that the temperature inside the feature monotonically increases with depth in stark contrast with the surrounding "cold collar" region where the temperature structure shows strong inversions (Section 3). Also inside the vortex eye the cloud top is located $\sim 7 \mathrm{~km}$ deeper than elsewhere on the planet (Fig. 15).

VIRTIS imaging in the near-IR $\mathrm{CO}_{2}$ absorption bands, whose relative depth is proportional to the cloud top pressure, provides an opportunity to map the cloud top altitude directly all over the globe. Figure 15 shows a VMC UV image with an overlaid colour mosaic of the cloud top altitude derived from simultaneous VIRTIS measurements in the $1.6 \mu \mathrm{m} \mathrm{CO} \mathrm{CO}_{2}$ band. The cloud top in the low- and mid-latitudes is located at $\sim 75 \mathrm{~km}$ (Titov et al., 2008). It begins descending at $\sim 55^{\circ} \mathrm{S}$ at 

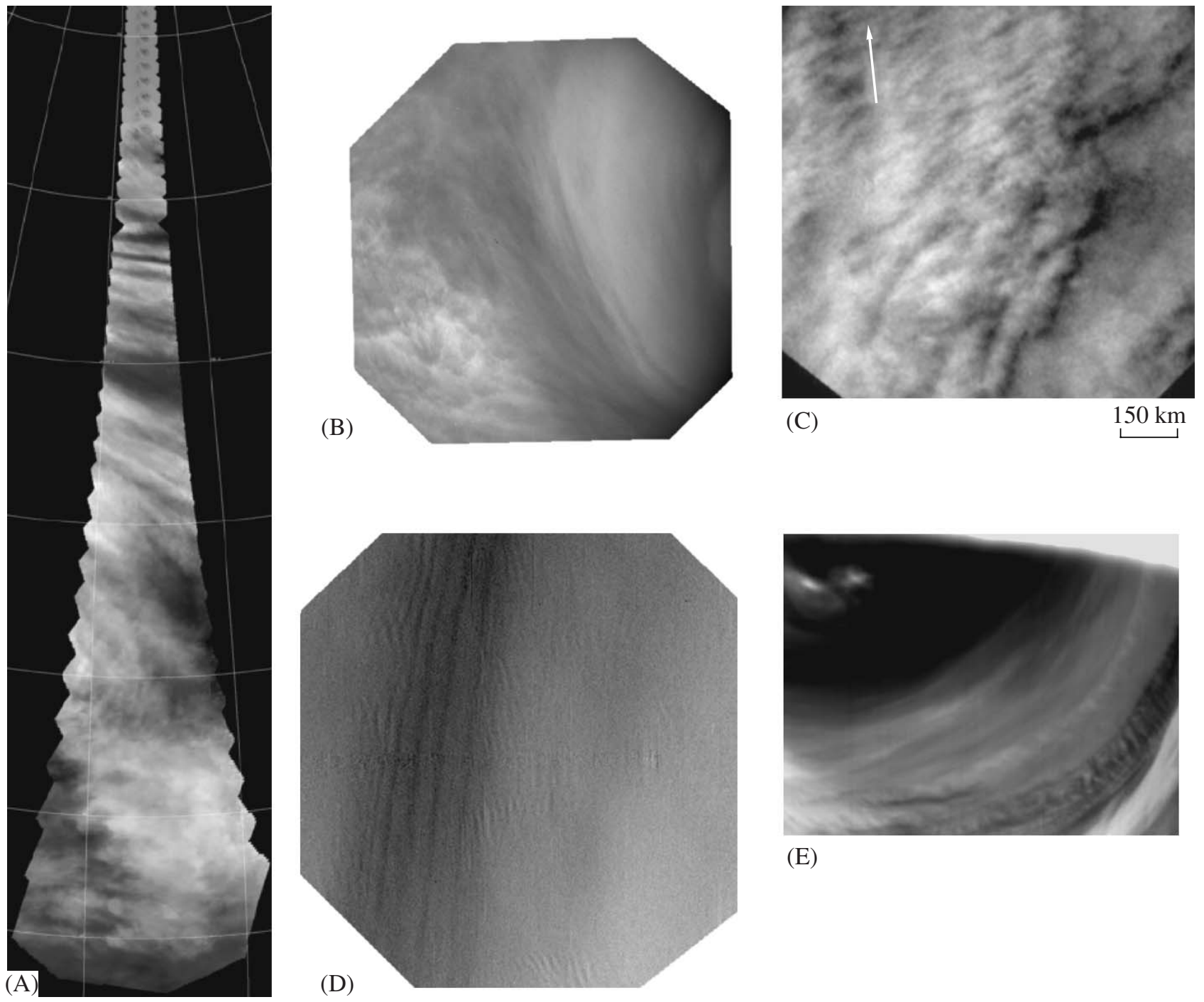

(E)

Fig. 13. Meso- and small-scale cloud morphology. (A) A mosaic composed of VMC UV images taken during pericentre pass in orbit no. 261. (B) VMC UV image of the mid-latitude transition region. (C) VMC high resolution UV image of the convective clouds at low latitudes. (D) Waves in the polar region $(\sim 70 \mathrm{~N})$ captured by the VMC UV channel. (E) Mid-latitude waves in the deep cloud seen by VIRTIS in the near-IR.

the edge of the cold collar and sinks as deep as $65 \mathrm{~km}$ in the vortex eye. Thus the cloud top altitude varies by more than a scale height over the planet. Surprisingly, the sharp outer boundary of the UV bright band is not evident in the cloud top maps, implying that both UV dark low latitude and bright mid-latitude clouds are located at about the same altitude level.

Limb observations and especially stellar occultation by SPICAV bring a wealth of information about the vertical structure and microphysical properties of the upper haze. VMC observed rather uniform structure of the aerosols at the limb. The limb brightness profiles are characterized by exponential decrease of brightness with very rare detached layers. More detailed investigations by SPICAV stellar occultation revealed upper haze in the altitude range $70-90 \mathrm{~km}$ with a scale height of 4-5 km. This is close to that of the gaseous atmosphere and allowed one to constrain the particle size distribution.

\section{ATMOSPHERIC DYNAMICS}

Early observations and models established that the Venus atmosphere has at least two dynamical regimes. The troposphere $(0-60 \mathrm{~km})$ and lower mesosphere up to $\sim 80 \mathrm{~km}$ are in the state of super-rotation: retrograde almost purely zonal motion with a wind speed that peaks at the cloud top and decreases down to the surface and above the clouds (Gierasch et al., 1997; Schubert et al., 2007). The observed global cloud pattern also shows obvious vortex organization of the cloud level dynamics (Fig. 12) The thermosphere (100-200 km) is 

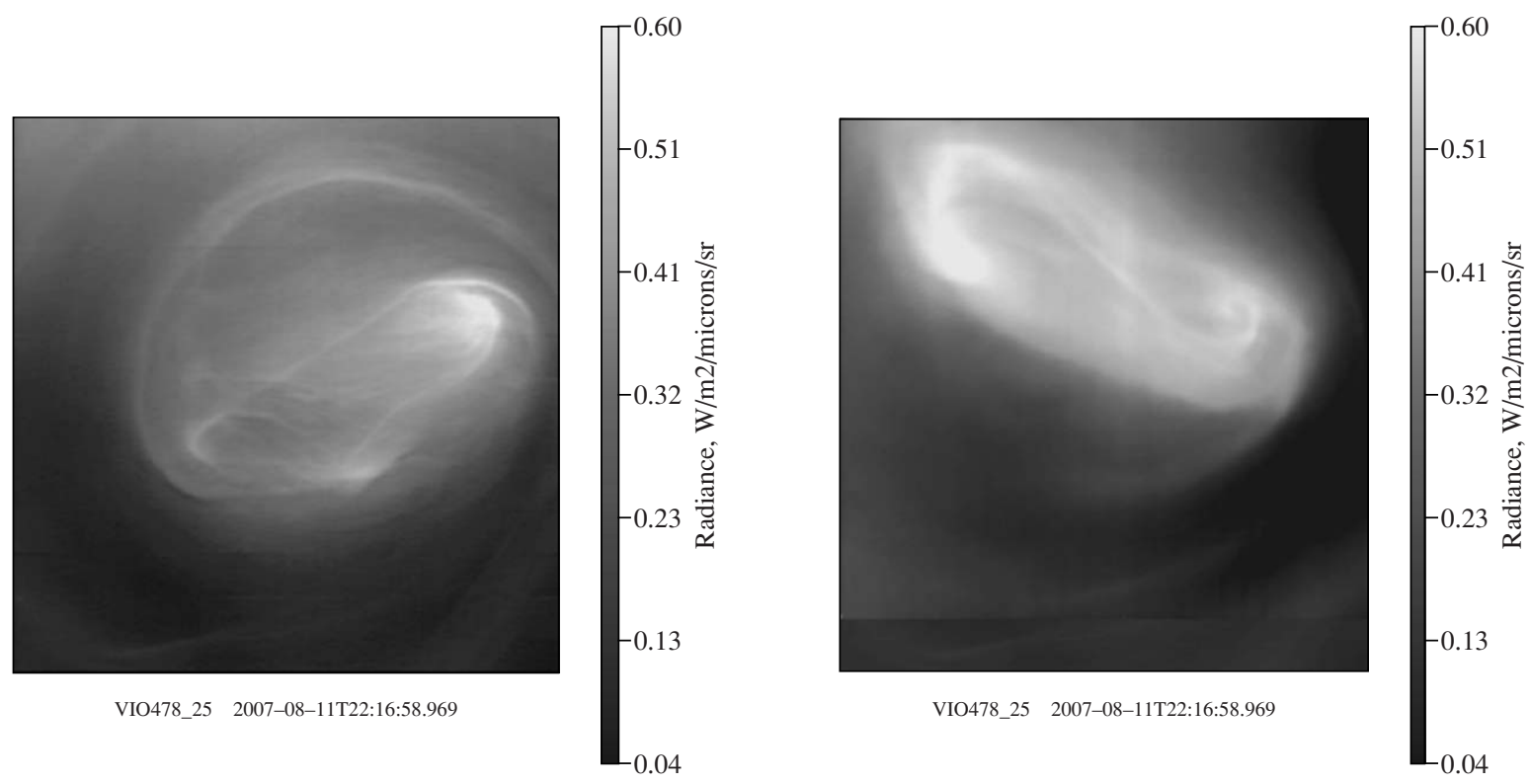

Fig. 14. Examples of high-resolution views of the vortex eye captured by VIRTIS at $5.1 \mu \mathrm{m}$.

involved in a global day-to-night circulation driven by higt temperature drop.

Spectro-imaging instruments onboard Venus Express observe atmospheric motions at altitudes from the cloud base $(\sim 50 \mathrm{~km})$ up to the lower mesosphere $(\sim 140 \mathrm{~km})$. The wind speeds are derived from tracking the motions of cloud features. Figure 16 shows the latitude profile of zonal wind at several altitudes derived from VIRTIS and VMC imaging (Sanchez-Lavega et al., 2008; Markiewicz et al., 2007b). At all heights the wind speeds within the cloud deck remain almost constant with latitude below $\sim 50^{\circ} \mathrm{S}$, but quickly fade out poleward of this latitude. Interestingly, this boundary coincides with the transition in cloud morphology (Fig. 12) and the outer edge of the "cold collar". Comparison to the thermal wind derived from the VIRTIS temperature sounding indicates that the cyclostrophic approximation works quite well, at least in the upper cloud region in middle and high latitudes (Fig. 16; Piccialli, et al., 2008). Preliminary studies suggest that zonal winds have a minimum in the late morning, increasing in the afternoon (Moissl, et al., 2009).

The altitude behaviour of the wind speed at low latitudes derived from Fig. 16 is in general agreement with the various descent probe measurements (Gierasch et al., 1997), keeping in mind the strong vertical wind shear, and the uncertainty in the altitude of the cloud markings used for wind tracking (Fig. 17). However, the cloud tracked winds are systematically slower than those derived from descent probes. The vertical wind shear at low latitudes is $3-5 \mathrm{~m} / \mathrm{s} / \mathrm{km}$, but vanishes in the middle and high latitudes. The Venus Express observations revealed this behaviour for the first time, since the descent probes covered only low latitudes.
Meridional winds are much weaker $(0-20 \mathrm{~m} / \mathrm{s})$ and more difficult to measure. They tend to increase from equator to mid-latitudes where the meridional wind speed reaches a maximum, and then decreases and even inverts its direction near the pole.

The Venus Express observations of the nightglow discussed in Section 5 provide important new clues about the thermospheric circulation. The distribution and morphology of the non-LTE emissions (Figs. 10, 11) (Gerard et al., 2008), as well as the temperature midnight maximum at the mesopause (Fig. 6) (Bertaux et al., 2007), provide indirect evidence for a solar-anti-solar circulation in the thermosphere. In addition, the sequences of nadir images taken in the non-LTE emissions bands monitor the apparent motions at mesopause levels $(\sim 100 \mathrm{~km})$ (Fig. 10b) for the first time. Despite difficulties interpreting $\mathrm{O}_{2}$ variability in terms of bulk velocities, Piccioni et al. (2007) made an attempt to derive the velocity field (Fig. 10b). The mean flow is dominated by meridional motions from polar latitudes equatorward and by a zonal flow from dawn to midnight, with apparent velocities of $\sim 60 \mathrm{~m} / \mathrm{s}$. The non-homogeneous, time-dependent distribution of the $\mathrm{O}_{2}\left({ }^{1} \Delta_{\mathrm{g}}\right)$ nightglow emission indicates that the local downward flow of oxygen may differ substantially from the mean value, in response to variations in factors such as the efficiency of the global day-night transport, the focusing effect of the night-side subsidence, changing zonal wind speeds, eddy transport efficiency, and gravity wave breaking. Figure 18 shows a sketch of the solaranti-solar circulation in the Venus thermosphere and explains physical mechanism responsible for the nonLTE emissions on the night side. 


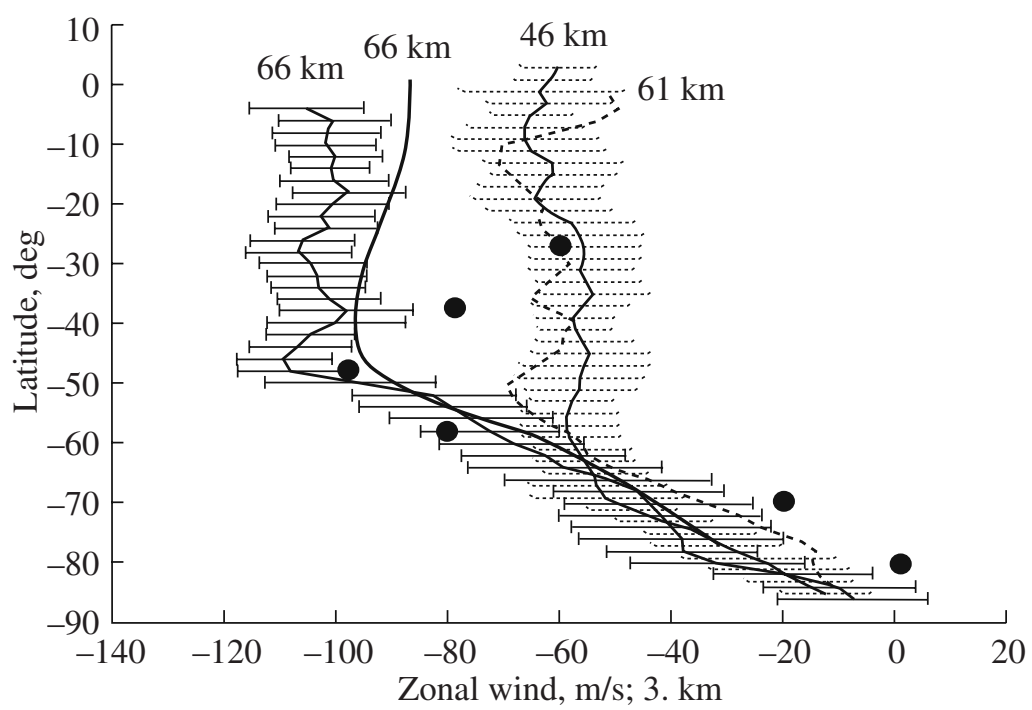

Fig. 16. Mean latitude profiles of the zonal wind speed in the Southern hemisphere derived from VIRTIS images at 66,61 aand $46 \mathrm{~km}$ and VMC UV images at $66 \mathrm{~km}$. Figures at the curves give the altitude with $\pm 2-3 \mathrm{~km}$ accuracy. Large black dots show cyclostrophic wind calculations for $\sim 66 \mathrm{~km}$ altitude based on the VIRTIS temperature sounding.

\section{PLASMA ENVIRONMENT}

The solar wind interaction with non-magnetized Venus results in the formation of an induced magnetosphere. In the induced magnetosphere the solar wind energy may be transferred to the planetary ions of the upper atmosphere resulting in acceleration, escape and loss. Therefore the investigation of the interaction processes is crucially important for understanding the evolution of the atmosphere. Venus Express investigates the circumplanetary plasma environment by means of three techniques. The VeRa experiment measures remotely vertical profiles of electron densities by radio occultation (Häusler et al., 2006), the double-sensor fluxgate magnetometer monitors the magnetic field (Zhang et al., 2006), and the ASPERA-4 experiment (Analyser of Space Plasmas and Energetic Atoms) measures insitu fluxes of energetic neutral atoms, ions and electrons (Barabash et al., 2007a). Venus Express measurements are taken at solar minimum, thus complementing the Pioneer Venus plasma studies that were acquired during solar maximum.

Venus Express passes through different regions and boundaries, namely bow shock, magnetosheath, and induced magnetosphere boundary, formed by interaction of the solar wind with the planet (Fig. 19), their boundaries clearly identified in the magnetic field and plasma measurements. Figure 20 shows an example of the magnetic field measurements taken on December 12, 2006. First the satellite crosses the bow shock on the day side where the magnetic field strength and wave power increase (point BS in Fig. 20). It then moves through the shocked plasma of the magnetosheath to the night side, crossing the induced magnetopause (IMB) and reaching the plasma sheet (PS) characterized by the change of the sign of $B_{x}$ component. Later, far in the magnetotail, it crosses the induced magnetosphere boundary and bow shock again. The size of the bow shock is largely determined by how completely the planetary obstacle deflects the solar wind. During April

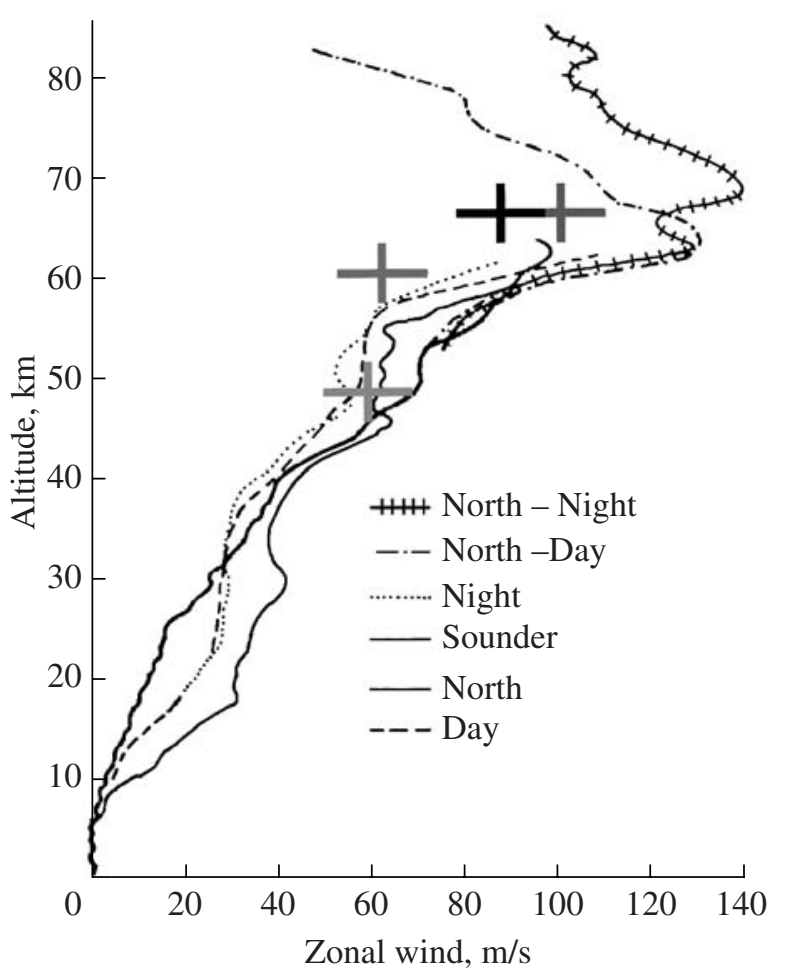

Fig. 17. Comparison of the equatorial zonal wind speeds derived from the VIRTIS and VMC Venus Express observations at different altitudes (Fig. 16) to the vertical profiles measured by descent probes (from Schubert, 1983). 


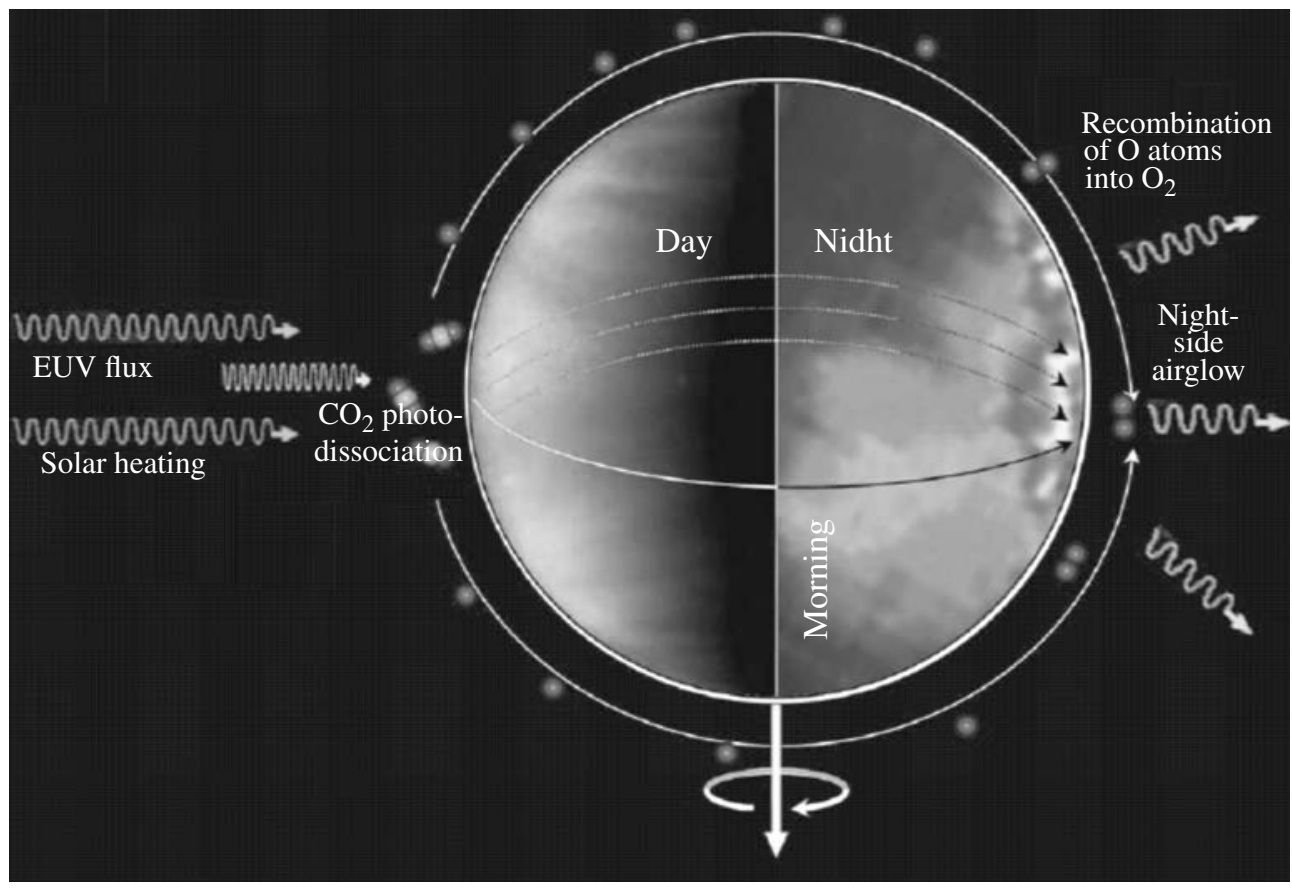

Fig. 18. Sketch of the solar-anti-solar global circulation pattern in the thermosphere of Venus $(>100 \mathrm{~km})$ that controls the night side airglow pattern (R. Hueso, J. Bailey).

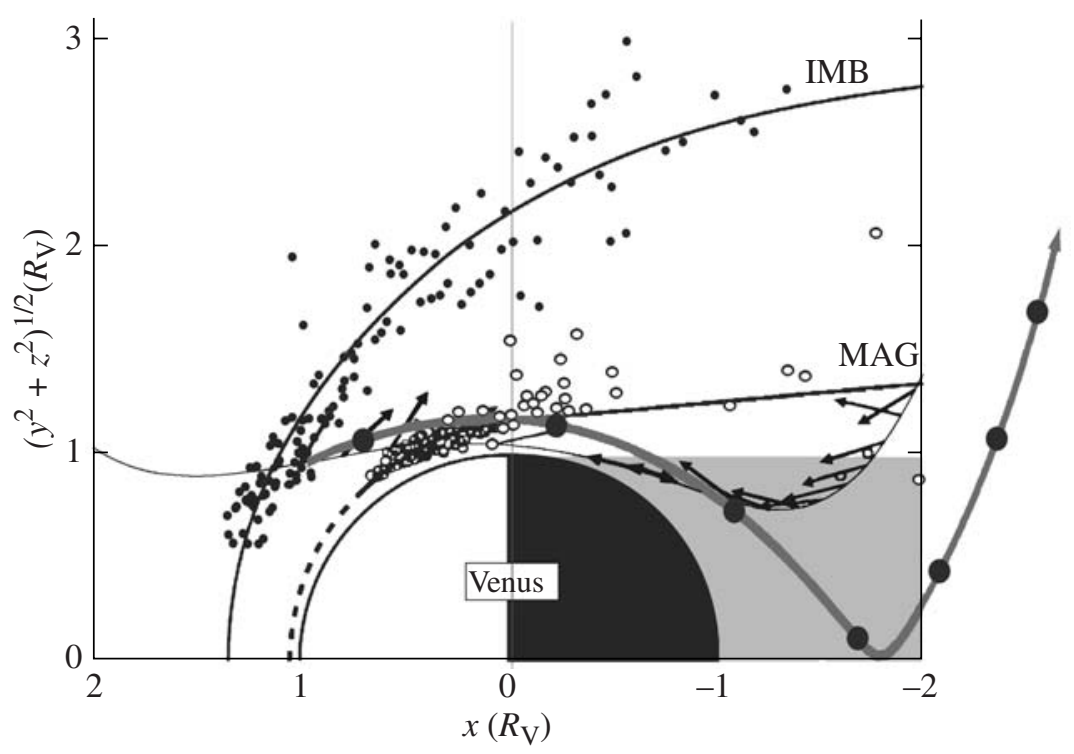

Fig. 19. Geometry of the bow shock (BS) and induced magnetopause boundary (IMB) derived from the MAG observations. Solid circles are the bow-shock crossings and the solid line is the best fit. The open circles are crossings of the magnetopause of the induced magnetosphere and the dashed-then-solid line is the best-fit model to these points. The shaded region is the optical shadow. $x$ is the direction to the Sun; $y$ is the direction opposite to planetary motion; and $z$ is northward, perpendicular to the orbit plane of Venus. The red curve shows the Venus Express orbit in these coordinates.

to August 2006, 147 clear crossings were obtained. The best fit to the bow shock location shown in Fig. 19 from a solar-zenith angle of $20^{\circ}$ to $120^{\circ}$ gives a terminator bow shock location of $2.14 R_{\mathrm{V}}$, which is $1600 \mathrm{~km}$ closer to Venus than the $2.4 R_{\mathrm{V}}$ measured at solar maximum, but somewhat further than reported by Venera 9 and 10 . 


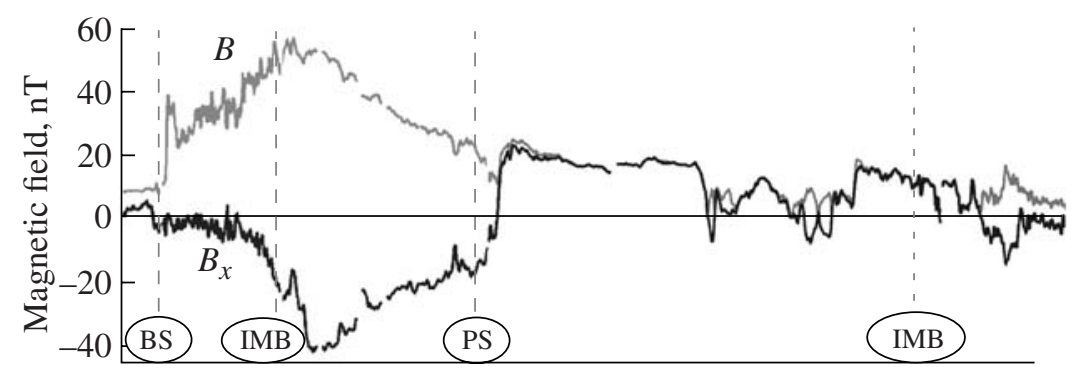

Fig. 20. Magnetic field measurements on December 12, 2006: $B$-absolute value, $B_{x}$-projection on the direction to the Sun.

The best fit to the subsolar bow shock is $1.32 R_{\mathrm{V}}$, only 1 $900 \mathrm{~km}$ above the surface of the planet. The observed bow shock seems to be in the position that would be expected from a complete deflection by a magnetized ionosphere. Thus the main conclusion is that little solar wind enters the Venus ionosphere even at solar minimum (Zhang et al., 2007).

The vertical structure of the ionosphere and its variations with latitude and local solar time were studied remotely by the radio occultation experiment VeRa. Figure 21 shows a collection of daytime electron density profiles (Pätzold et al., 2007). Two layers, $\mathrm{V}_{1}$ and $\mathrm{V}_{2}$, are generated by photoionization of $\mathrm{CO}_{2}$ by solar extreme-ultraviolet and soft X-rays. The structure above $\sim 180 \mathrm{~km}\left(\mathrm{~V}_{3}\right)$ is presumably controlled by the $\mathrm{O}^{+}$ ion. The majority of electron density profiles display a bulge in the topside between 160 and $180 \mathrm{~km}$ that is not documented in theoretical models of ion and electron production. Large density variations can be seen in the topside ionosphere $\left(\mathrm{V}_{3}\right)$ while the lower $\mathrm{V}_{1}$ and $\mathrm{V}_{2}$ layers are relatively stable. Electron density peaks at $\sim 4 \times$ $10^{5} \mathrm{~cm}^{-3}$ at about $140 \mathrm{~km}$. Ionization completely disappears by $\sim 120 \mathrm{~km}$. On the night side the electron density abruptly drops by 1-2 orders of magnitude behind terminator.

The ASPERA-4 experiment has established for the first time the composition of escaping plasma (Barabash et al, 2007b). It consists mostly of $\mathrm{O}^{+}$and $\mathrm{H}^{+}$with some admixture of $\mathrm{He}^{+}$(Fig. 22). ASPERA-4 also characterized the energy distribution of the escaping planetary ions (Fig. 22) and reveals basic acceleration processes. At Venus, ion acceleration is produced by three mechanisms: by ion pick-up (that is, by acceleration in the convection electric field), by instabilities at the induced magnetosphere boundary resulting in detached plasma clouds, and by polarization electric fields at low altitudes in the night-side ionosphere where the field is nearly radial. ASPERA observed two different populations of the accelerated planetary ions, namely ions escaping through the plasma sheet, and ions escaping through the induced magnetosphere boundary layer (Fig. 22). The energy distributions of these two populations are different. The ratio of the $\mathrm{O}^{+} / \mathrm{He}^{+} / \mathrm{H}^{+}$energies in the plasma sheet is about 4:2:1 (Fig. 22c). The ion energy is therefore a function of mass, suggesting that ion pick-up is an acceleration mechanism in the plasma sheet, in general agreement with the previous simulations for $\mathrm{O}^{+}$. However, ordinary pick-up would result in a 16:4:1 ratio. The difference may be due to speciesdependent assimilation into the plasma flow.

The ion escape considered here occurs through two main regions: the plasma sheet and a boundary layer at the induced magnetosphere boundary (Fig. 23). As expected, the alignment of the plasma sheet plane and the ion energy are controlled by the electric field. All

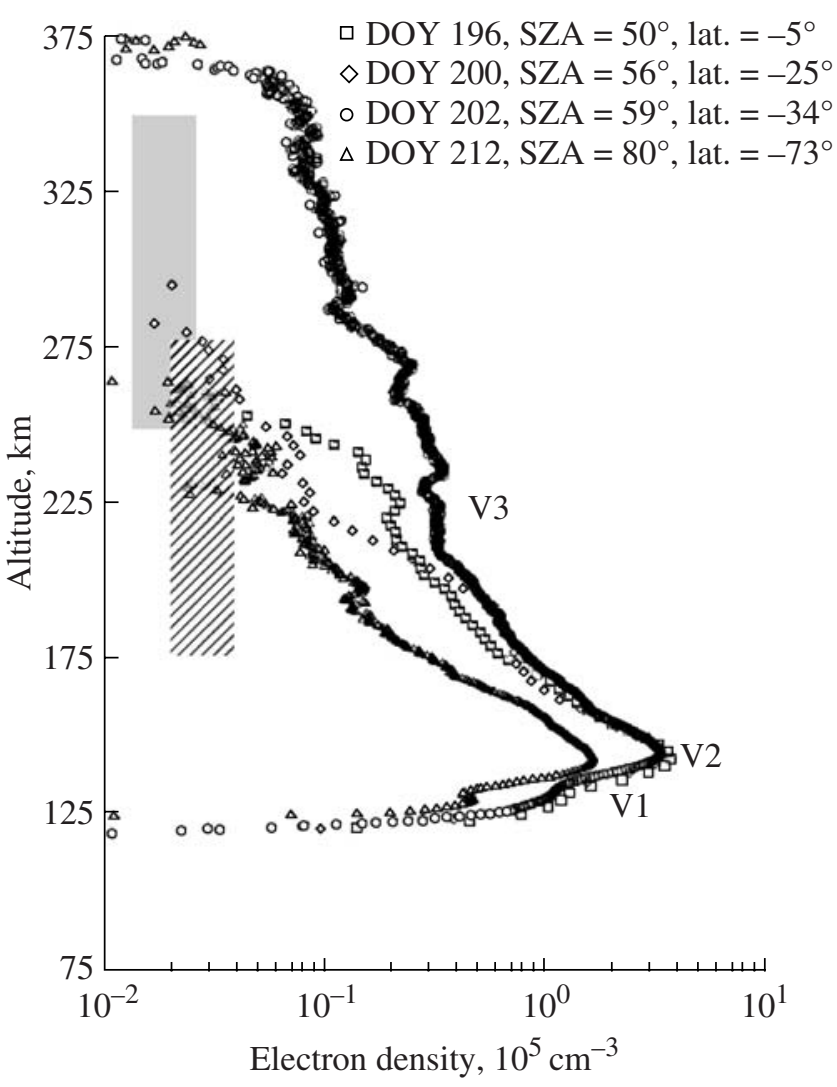

Fig. 21. Vertical profiles of electron density at different solar zenith angles retrieved from radio occultation soundings by VeRa. The shaded area shows pericentre altitude in the nominal mission, and the hatched area the pericentre "corridor" after its planned lowering in summer 2008. 
(a)

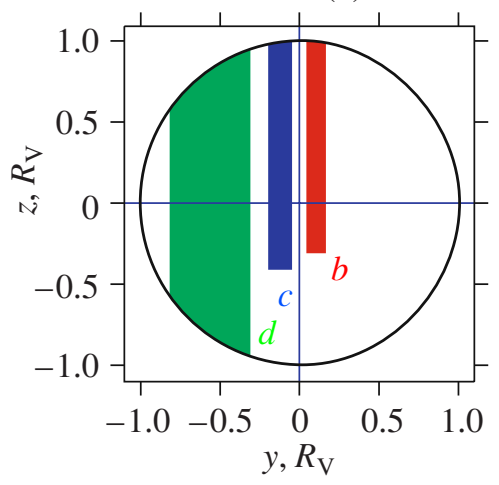

(c)

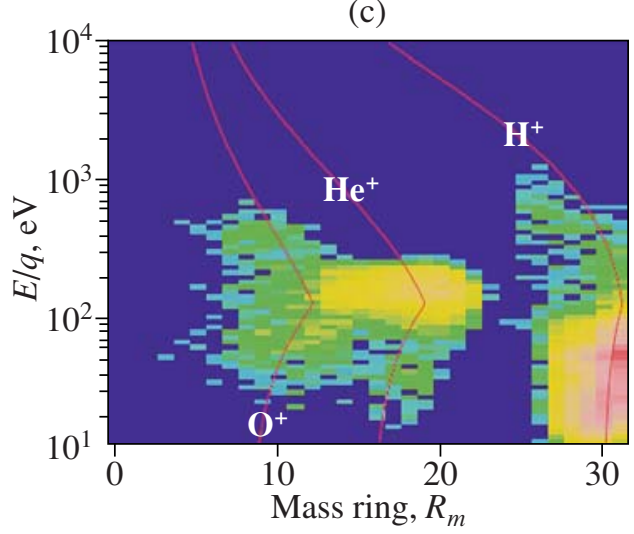

(b)
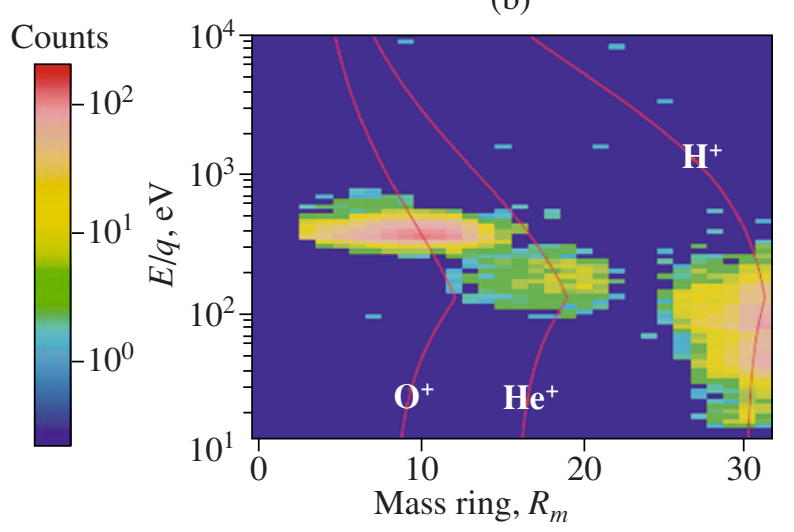

(d)

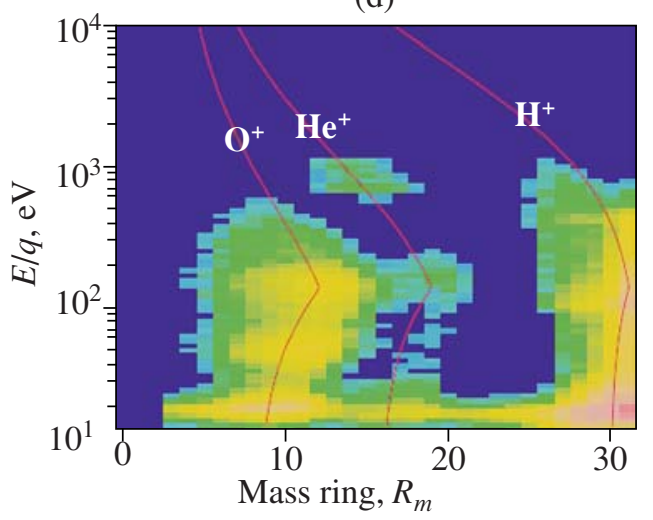

Fig. 22. Composition and energy distribution of the escaping plasma. (a) Geometry of the three spatial regions where the energymass matrixes were collected in the Vse (Venus-solar-electrical) coordinate system in which the Z-axis is co-aligned with the convectional electrical field. (b)-(d) The energy-mass matrixes accumulated over 33 orbits during the period from May 18 through December 30, 2006 for the $+Y$ lobe (b), $-Y$ lobe (c) and IMB layer (d). Vertical axes are energy/charge $(E / q)$ ratio, the horizontal axes are the position of the ion impact on the detector (a sensor mass ring, $R_{m}$ ), and the colour codes the counts that were accumulated over all directions and averaged over occurrences. The red lines represent constant mass.

three species show similar spatial distributions, confirming that both $\mathrm{H}^{+}$and $\mathrm{He}^{+}$are indeed of planetary rather than solar-wind origin. The escape rates are obtained by integrating the measured flux over the plane perpendicular to the $X$ axis. Although the spatial coverage is still not sufficient to obtain the total average escape rate, one can obtain reliable flux ratios. These are $\mathrm{Q}\left(\mathrm{H}^{+}\right) / \mathrm{Q}\left(\mathrm{O}^{+}\right)=1.9$ and $\mathrm{Q}\left(\mathrm{He}^{+}\right) / \mathrm{Q}\left(\mathrm{O}^{+}\right)=0.07$.

The measured composition differs significantly from the plasma composition at the ionopause altitude $(300 \mathrm{~km}$ ) for solar minimum conditions (Fox, 2004), where the respective number-density ratios are $n\left(\mathrm{H}^{+}\right) / n\left(\mathrm{O}^{+}\right)=0.1$ and $n\left(\mathrm{He}^{+}\right) / n\left(\mathrm{O}^{+}\right)=4 \times 10^{-3}$. The observed enrichment of escaping plasma in light species may result from two causes. First, the ion pick-up process operating primarily at higher altitudes, 600$700 \mathrm{~km}$, in which the densities of light species are increased with respect to heavier ions. Second, acceleration by the polarization electric field increasing the flux of lighter ions with respect to heavier, because lighter ions gain higher velocities for the same energy.
The ratio of the escape rates of hydrogen and oxygen is a critical parameter in understanding both the dryness and the oxidation state of the Venusian atmosphere. The measured ratios concern only ions and do not include the escape of the respective atoms. Scaling the measured ratio $\mathrm{Q}\left(\mathrm{H}^{+}\right) / \mathrm{Q}\left(\mathrm{O}^{+}\right)=1.9$ to account for the neutral losses, we arrive at a ratio for the total (neutral atoms plus ions) loss of 2.2:1, very close to the stoichiometric value of 2:1 for the water molecule. Some excess of $\mathrm{H}^{+}$could be due to solar wind protons, which may penetrate through the induced magnetosphere boundary in small amounts and contribute to the measured $\mathrm{H}^{+}$flux in the boundary layer. That the present escape of hydrogen and oxygen through the wake takes place in the stoichiometric ratio of water implies that the atmosphere of Venus did not change its oxidation state after steady state conditions had been reached, in contrast with that of Mars (Lammer et al., 2003). This result is thus consistent with the suggestion from atmospheric and ionospheric observations by the Pioneer Venus orbiter (McElroy et al., 1982) that Venus loses 

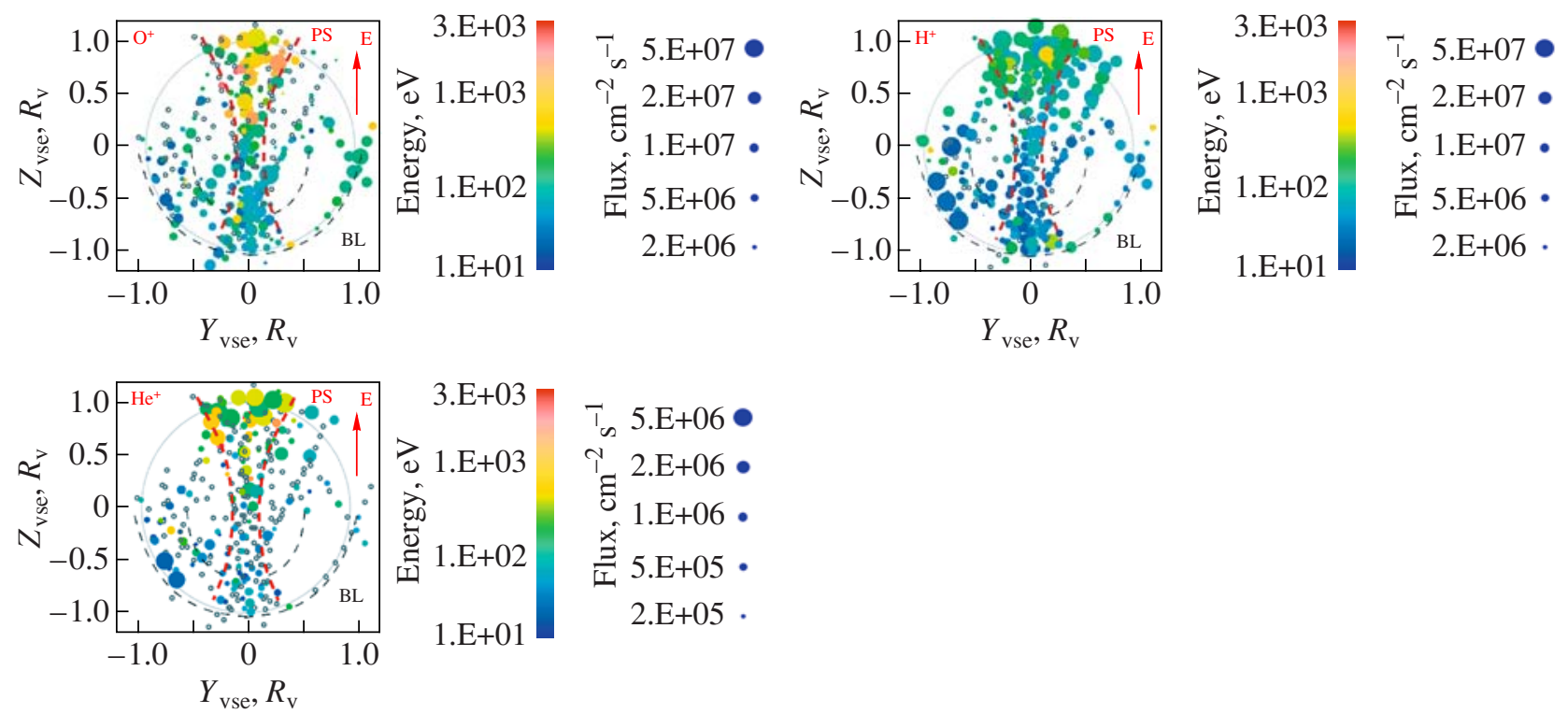

Fig. 23. Spatial distribution of the escaping plasma. The measured $\mathrm{O}^{+}, \mathrm{He}^{+}, \mathrm{H}^{+}$flux distributions in the tail region from 33 orbits were integrated over $X_{\mathrm{vse}}[-0.5,-3.0]$ and are shown in a $Y_{\mathrm{vse}}-Z_{\mathrm{vse}}$ plane across the tail. Venus' geometrical eclipse is shown by the thin grey circle. To ensure that no solar wind protons affect the mass composition measurements inside the IMB, we restrict the area of the analysis to $R<1.2 R_{\mathrm{V}}$. Blank circles show measurements with zero flux. The flux scale for $\mathrm{He}^{+}$is 10 times lower than those for $\mathrm{O}^{+}$and $\mathrm{H}^{+}$. The plasma sheet region is identified by red dashed lines and marked "PS", the boundary layer at the IMB by black dashed lines and marked "BL", while the convection electric field direction is marked "E".

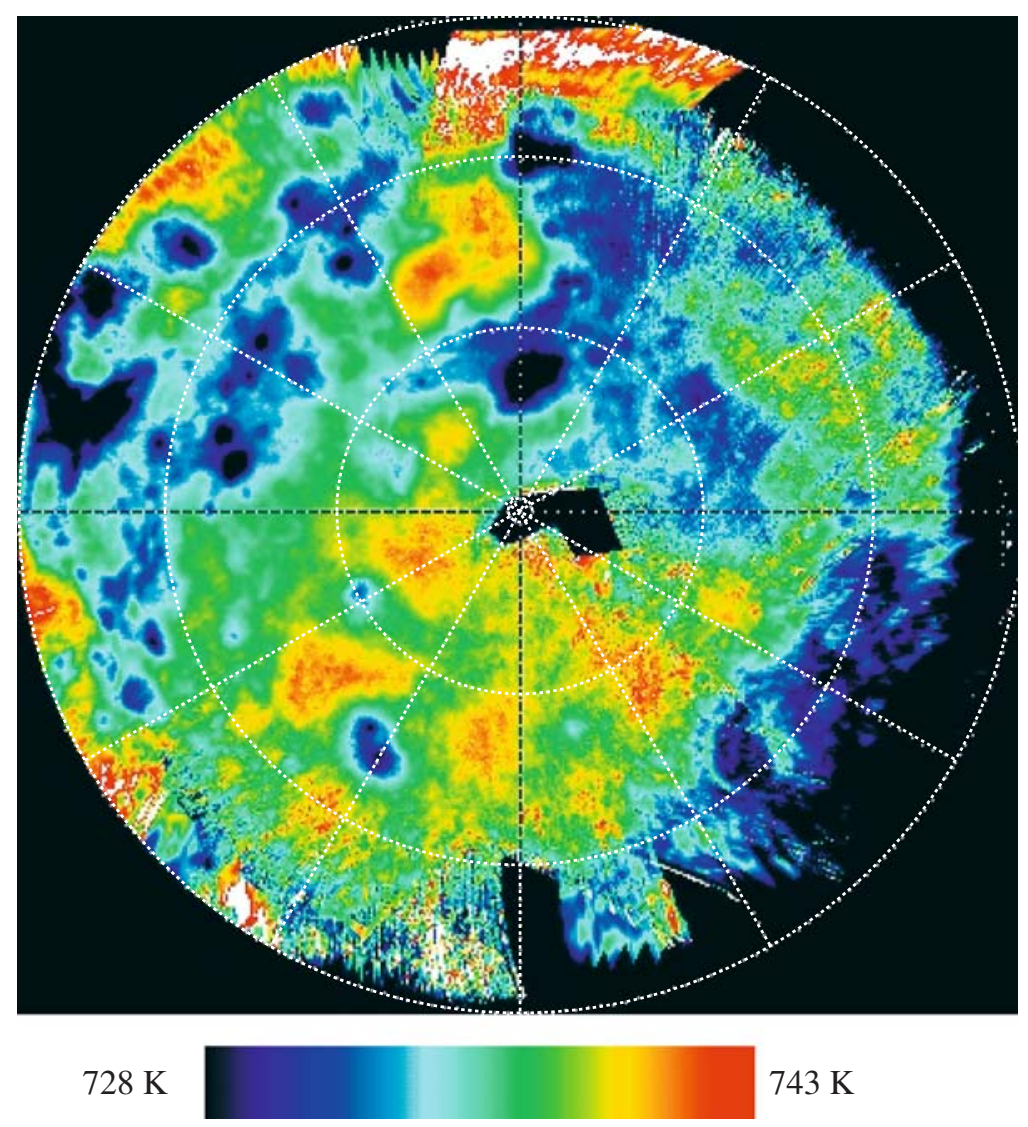

Fig. 24. VIRTIS thermal map of the Venus Southern hemisphere. 


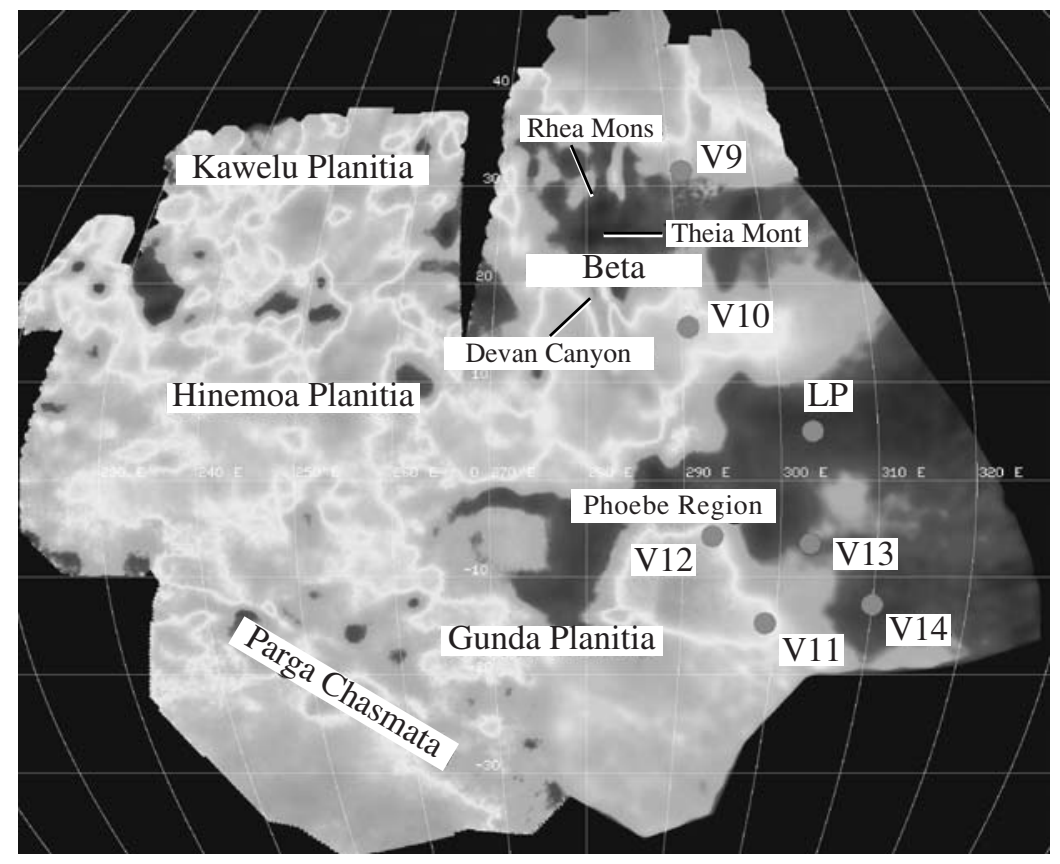

Fig. 25. VMC thermal map of Beta and Phoebe regions and Hinemoa Planitia. Dots show Venera (V-9, -14) and Pioneer Venus Large Probe (LP) landing sites.

the constituents of water in a ratio that maintains the system's oxidation state. The absolute escape rates, including the implications for long-term water evolution, will be determined later.

The observed relative abundance of $\mathrm{He}^{+}$in the escaping plasma from Venus is surprisingly high. This may be due to the more effective acceleration caused by the polarization field. The higher pressure gradients associated with the stronger gravitational field on Venus results in a higher polarization field that operates over larger distances at Venus than at Mars. Thus, whereas the stronger gravitational field of Venus reduces the Jeans escape of the heavier main constituents of the atmosphere, almost paradoxically it facilitates the loss of the lighter species.

The main result of these observations, however, is the establishment of potentially important ion escape from present-day Venus through the plasma wake region. Further observations in this region as the solar cycle progresses towards maximum will establish the variability of these escape rates and will improve our ability to infer the history of Venus's water.

\section{SURFACE INVESTIGATIONS}

Although Venus Express has no instrument specifically dedicated to surface studies, its payload still can contribute to this topic by several techniques (Titov et al, 2006b; Svedhem et al., 2007a). The first one is the bistatic radar sounding by VeRa (Häusler et al., 2006), in which the spacecraft high-gain antenna tracks a selected surface target during the pericentre pass and the signal reflected by the surface is received by ground stations. Nine bi-static radar soundings were performed during the nominal mission over the surface features known as Maxwell, Theia, Rhea, and Ozza Montes. In the second method, accurate tracking of the spacecraft during the pericentre pass allows one to study variations of the Venus gravity field created by inhomogeneities of mass distribution in the solid body of the planet. Several experiments of this kind were performed over Atalanta Planitia. Unfortunate loss of the S-band signal in the spacecraft telecommunication system led to the termination of both experiments in summer 2007.

For the first time Venus Express has carried out systematic thermal mapping of the surface in the $1 \mu \mathrm{m}$ transparency window on the night side. VIRTIS acquires mosaics of the Southern hemisphere from apocentre and the ascending branch of the orbit (Müller et al., 2007). VMC takes close-up images of the equatorial region when the spacecraft is in eclipse, so the observations are not polluted by stray light (Markiewicz et al., 2007a). Figure 24 shows a thermal mosaic of the Southern hemisphere obtained by VIRTIS during the nominal mission (Mueller et al, 2008). Brightness contrasts observed in the $1 \mu \mathrm{m}$ night-side images are caused by variations in surface temperature and to lesser extent its emissivity, as well as a large contribution from the overlying cloud opacity, which must be removed to reveal the surface temperature. The surface is assumed to be in thermal equilibrium with the atmosphere so its temperature follows the topography, with higher regions progressively colder according to the atmospheric lapse rate. Thus major relief features are 
clearly recognized in the map in Fig. 24, although the spatial resolution is severely degraded by multiple scattering in the thick atmosphere to $\sim 50 \mathrm{~km}$ at best. Figure 25 shows similar map created from the VMC night side images taken during two eclipse seasons in MarchApril and July-August 2007. They cover Hinemoa Planitia, and Beta and Phoebe Regio, the eastern flanks of which were visited by several Venera and Pioneer Venus descent probes in 1970-1980s.

The main goals of surface thermal mapping are to search for active volcanism and to identify correlations between emissivity anomalies and the geological units seen in the Magellan radar data. Interpretation of the thermal maps like those shown in Figs. 24 and 25 is a challenging task since the surface is observed through the cloud deck with opacity of $\sim 20-40$. Removal of the cloud contribution is achieved by VIRTIS using simultaneous imaging in the $1.31 \mu \mathrm{m}$ window, which has major contribution from the clouds. Analysis of the VMC images needs additional data or assumptions.

Preliminary results show that surface emissivity variations derived from the VIRTIS maps (Fig. 24) reach about $10 \%$. In some places the contacts between the units that are bright and dark in radar maps are not visible in the VMC images, suggesting that the mineralogical composition of their surface materials, their porosity and the micron-to-mm texture are all approximately the same. Visual inspection of the VMC images of the potentially volcanically active areas in Beta and Phoebe Regio so far have not revealed any sign of lava flows large enough and hot enough to be detected.

\section{SUMMARY OF THE RESULTS}

The Venus Express observations during the nominal mission brought a rich harvest of scientific results covering virtually all aspects of the physics of the Earth's mysterious neighbour (Svedhem et al., 2007b). The observations of cloud morphology and wind field covering a range of altitudes within the cloud layer at high spatial resolution revealed global vortex-type organization of the atmospheric circulation. The equatorial atmosphere at the tropopause $(\sim 60 \mathrm{~km})$ is governed by convective and wave-dominated meteorology, with an abrupt transition to smoother "laminar" flow at 50 degrees latitude coinciding with the outer edge of the global bright UV band (Fig. 12). From the earlier thermal IR observations this region is known as the "cold collar". It encloses the eye of the global vortex - a vast oval structure several thousand kilometres across that rotates every 2.5-2.8 Earth days. The cloud top here is located $\sim 5 \mathrm{~km}$ lower than elsewhere on the planet, implying strong descending flow. The wind speeds derived from tracking the motions of cloud features were found to be in agreement with the cyclostrophic approximation, at least in the middle and high latitudes. Night-side observations in the transparent spectral "windows" showed that the vortex structure and circulation exist down to as least as great a depth as the lower cloud deck at 50-
$55 \mathrm{~km}$. The edge of the cold collar at $50-60^{\circ}$ latitude apparently marks the poleward limit of the Hadley circulation, the planet-wide overturning of the atmosphere in response to the concentration of solar heating in the equatorial zones. Indirect evidence of such meridional circulation is provided by monitoring of the latitude distribution of minor constituents, especially carbon monoxide, as dynamical tracers in the lower atmosphere. Comparison of the Venus Express observations with the results of the earlier missions convincingly proved hemispheric symmetry of the global circulation pattern.

The mesopause on Venus at $100-120 \mathrm{~km}$ altitude marks a transition between different global circulation regimes. The predominance of zonal super-rotation in the lower atmosphere below the mesopause is replaced by solar to anti-solar flow in the thermosphere above. Venus Express provided important clues about the upper atmosphere circulation. The non-LTE emission in the $\mathrm{O}_{2}$ band at $1.27 \mu \mathrm{m}$ that originates from the recombination of oxygen atoms on the night side peaks at around midnight (Fig. 11) (Drossart et al., 2007b; Gerard et al., 2008). Also the temperature maximum observed on the night side was attributed to adiabatic heating in the subsiding branch of the thermospheric solar to anti-solar circulation (Fig. 6b) (Bertaux et al., 2007b).

Full exploitation of new observational techniques like solar occultation and spectroscopy in the spectral windows on the night side allowed Venus Express to make a breakthrough in composition studies. Global surveys of abundance of trace gases $\left(\mathrm{CO}, \mathrm{COS}, \mathrm{H}_{2} \mathrm{O}\right)$ in the lower atmosphere (Fig. 9) provide important new constraints on chemical and dynamical models. Simultaneous measurements of the vertical profiles of hydrogen-bearing species in the upper atmosphere (Fig. 8, Bertaux et al., 2007b) and in situ monitoring of plasma properties (Barabash et al., 2007b) have begun to characterize the escape processes responsible for the depletion of water on Venus over the planet's history. Observations above the clouds uncovered a strong variability in both $\mathrm{H}_{2} \mathrm{O}$ and $\mathrm{HDO}$ content, and revealed a D/H ratio of $\sim 250$ - about twice as high as reported by earlier measurements in the lower atmosphere (Donahue and Russell, 1997). This unexpected result was tentatively explained by isotope fractionation in the process of ice particle condensation and atmospheric transport (Bertaux et al., 2007). This implies that species bearing hydrogen and deuterium are already fractionated in the mesosphere just below the region from which escape occurs.

Analyzer of Space Plasmas and Energetic Atoms (ASPERA-4) has established the composition of escaping planetary ions for the first time (Barabash et al., 2007b). After $\mathrm{H}^{+}$, the main escaping ion is $\mathrm{O}^{+}$. This is in contrast with Mars, where the escaping plasma consists of approximately equal amounts of $\mathrm{O}^{+}, \mathrm{O}_{2}^{+}$and $20 \% \mathrm{CO}_{2}$. The difference results from the higher gravitational acceleration at Venus, which tends to retain 
heavier components such as $\mathrm{CO}_{2}$. High fluxes of escaping $\mathrm{He}^{+}$were also detected. Interestingly $\mathrm{H}^{+}$and $\mathrm{O}^{+}$ ions were found to escape in roughly the $2: 1$ stoichiometric ratio found in the water molecule. Oxygen and hydrogen ions are formed by the dissociation of neutral atmospheric species by solar ultraviolet radiation, charge exchange, electron impact and other ionization processes, gain energy from the solar wind, and then escape through the planet's wake. These loss processes may have removed large amounts of water from Venus during the first billion years or so after the formation of the Solar System. A detailed quantification of the loss rates enables a more accurate estimate of how much water Venus has lost over its entire history, and by the end of the mission we should know better whether the planet once had an ocean as extensive and deep as Earth's.

For a long time the existence of lightning on Venus has been controversial. Whistler-mode waves, which can be considered reliable evidence of lightning, were detected by the Venus Express magnetometer during more than $10 \%$ of the pericentre passes (Russell et al, 2007). This corresponds to a lightning rate equal to at least half of that on Earth. Frequent lightning represents a significant energy input that has important implications for the chemistry in the lower and middle atmosphere on Earth, and this now seems likely to be true for Venus also.

The overall sense of the results of the Venus Express nominal mission is that the differences, particularly in climate, between Venus and Earth are much less mysterious than previously thought after the early phase of spacecraft exploration. They are consistent with theoretical ideas and interpretations suggesting that the two planets had similar surface environments in the past and that they evolved differently, with Earth's oceans converting most of its atmospheric $\mathrm{CO}_{2}$ to carbonate rocks, and Venus losing most of its water to space because of high surface temperature due to proximity to the Sun. Both processes can now be seen to be still going on. The high zonal winds and near-equatorial turbulence on Venus, as well of course as the high surface temperatures, result from the depth of the atmosphere and huge inventory of greenhouse gas retained by Venus. The slow rotation of Venus, as well as possibly being responsible for the lack of magnetic field that makes erosion of the atmosphere by the solar wind different from the Earth, in particularly, in the past, permits the Earth-like Hadley cell component of the atmospheric circulation to extend closer to the poles, where it breaks down in spectacular fashion to form midlatitude jets and polar vortices that are larger and more energetic than Earth's but are in some respects quite similar.

\section{PLANS FOR EXTENDED MISSION}

The nominal mission demonstrated that Venus Express is an excellent combination of a flexible space- craft, capable payload, and efficient ground segment. The first mission extension has been approved until the end of 2009, allowing Venus Express to extend the spatial and temporal coverage of the planet to carry out detailed observations of the phenomena discovered during the nominal mission. New modes of the spacecraft science operations can also be implemented, such as spot pointing to keep a selected target in the field of view of the instruments for longer time and to observe it at a varying angle. When applied to limb tracking this provides longer observations of the limb. Finally, a nadir "pendulum" mode allows the instruments to extend the duration of dayside observations while staying within allowed thermal conditions.

In July-August 2008 the pericentre altitude was reduced from $250-350$ to $180-280 \mathrm{~km}$. This allowed ASPERA and MAG to continue plasma and magnetic field investigations at lower altitude and move the investigated region closer to that remotely sounded by SPICAV and VeRa in occultation geometry (Fig. 21). Further pericentre lowering planned in autumn of 2009 would allow to carry out orbital decay experimental, based on accurate monitiring of the orbital parameters to derive the structure of the middle thermosphere above the Northern pole, a region never studied before. Moving closer to the planet in pericentre will also provide better conditions for the gravity experiment.

The pericentre lowering is now seen as only the first stage of orbit modification. Deeper diving into the atmosphere would enable an atmospheric drag experiment (VEXADE), in which the spacecraft deceleration due to atmospheric drag will be directly measured by onboard accelerometers to study the structure of the thermosphere and its variations. In the more distant future the experience gained in these operations can be used for aerobraking, with the eventual goal to bring the apocentre closer to the planet. The low eccentricity orbit will be more stable with respect to the solar gravity perturbations, resulting in longer duration for the mission. Also, the imaging instruments will be able to observe the Southern hemisphere at higher resolution.

The available onboard resources can keep Venus Express operational till 2013. During this time, the arrival of the Japanese Planet-C orbiter at Venus in December 2010 (Nakamura et al., 2007) will open broad opportunities for joint observation campaigns by two spacecraft.

\section{ACKNOWLEDGMENTS}

The authors are grateful to colleagues in the experimental teams, at EADS-Astrium, ESOC, ESTEC and ESAC, who contributed to the very successful Venus Express nominal mission. We also thank the colleagues who provided unpublished data for this paper. 


\section{REFERENCES}

1. Allen, D.A. and Crawford, J.W., Cloud Structure on the Dark Side of Venus, Nature, 1984, vol. 307, pp. 508511.

2. Barabash, S., Sauvaud, J.-A., Gunell, H., Andersson, H., Brinkfeldt, K., Grigoriev, A., Brinkfeldt, K., Holmstroem, M., Lundin, R., Yamauchi, M., Asamura, K., Baumjohann, W., Zhang, T.L., Coates, A.J., Linder, D.R., Kataria, D.O., Curtis, C.C., Hsieh, K.C., Sandel, B.R., Fedorov, A., Mazelle, C., Thocaven, J.-J., Grande, M., Koskinen, H.E.J., Kallio, E., Saeles, T., Riihela, P., Kozyra, J., Krupp, N., Woch, J., Luhmann, J., McKennaLawlor, S., Orsini, S., Cerulli-Irelli, R., Mura, M., Milillo, M., Maggi, M., Roelof, E., Brandt, P., Russell, C.T., Szego, K., Winningham, J.D., Frahm, R.A., Scherrer, J., Sharber, J.R., Wurz, P., and Bochsler, P., The Analyzer of Space Plasmas and Energetic Atoms (ASPERA-4) for the Venus Express mission, Planet. Space Sci., 2007a, vol. 55, pp. 1772-1792.

3. Barabash, S., Fedorov, A., Sauvaud, J.J., Lundin, R., Russell, C.T., Futaana, Y., Zhang, T.L., Andersson, H., Brinkfeldt, K., Grigoriev, A., Holmstroem, M., Yamauchi, M., Asamura, K., Baumjohann, W., Lammer, H., Coates, A.J., Kataria, D.O., Linder, D.R., Curtis, C.C., Hsieh, K.C., Sandel, B.R., Grande, M., Gunell, H., Koskinen, H.E.J., Kallio, E., Riihela, P., Saeles, T., Schmidt, W., Kozyra, J., Krupp, N., Fraenz, M., Woch, J., Luhmann, J., McKenna-Lawlor, S., Mazelle, C., Thocaven, J.-J., Orsini, S., Cerulli-Irelli, R., Mura, M., Milillo, M., Maggi, M., Roelof, E., Brandt, P., Szego, K., Winningham, J.D., Frahm, R.A., Scherrer, J., Sharber, J.R., Wurz, P., and Bochsler, P., The Loss of Ions from Venus Through the Plasma Wake, Nature, 2007b, vol. 450, pp. 650-653.

4. Barabash, S. et al., The Analyzer of Space Plasma and Energetic Atoms (ASPERA-4) for the Venus Express Mission, ESA SP-1295, The Netherlands, Noordwijk, 2008 (in press).

5. Bertaux, J.-L., Nevejans, D., Korablev, O., Villard, E., Quemerais, E., Neefs, E., Montmessin, F., Leblanc, F., Dubois, J.P., Dimarellis, E., Hauchecorne, A., Lefevre, F., Rannou, P., Chaufray, J.Y., Cabane, M., Cernogora, G., Souchon, G., Semelin, F., Reberac, A., Van Ransbeek, E., Berkenbosch, S., Clairquin, R., Muller, C., Forget, F., Hourdin, F., Talagrand, O., Rodin, A., Fedorova, A., Stepanov, A., Vinogradov, I., Kiselev, A., Kalinnikov, Yu., Durry, G., Sandel, B., Stern, A., and Gerard, J.C., SPICAV/SOIR on Venus Express: Three Spectrometers to Study the Global Structure and Composition of the Venus Atmosphere, Planet. Space Sci., 2007a, vol. 55, pp. 1673-1700.

6. Bertaux, J.-L., Vandaele, A.-C., Korablev, O., Villard, E., Fedorova, A., Fussen, D., Quemerais, E., Belyaev, D., Mahieux, A., Montmessin, F., Muller, C., Neefs, E., Nevejans, D., Wilquet, V., Dubois, J.P., Hauchecorne, A., Stepanov, A., Vinogradov, I., and Rodin, A., A Warm Layer in Venus' Cryosphere and High-Altitude Measurements of $\mathrm{HF}, \mathrm{HCl}, \mathrm{H}_{2} \mathrm{O}$ and $\mathrm{HDO}$, Nature, 2007b, vol. 450, pp. 646-649.

7. Bertaux, J.-L., Nevejans, D., Korablev, O., Villard, E., Quemerais, E., Montmessin, F., Leblanc, F., Chassefiere, E., Dubois, J.P., Dimarellis, E., Hauchecorne, A., Lefevre, F., Rannou, P., Cabane, M., Cernogora, G.,
Souchon, G., Semelin, F., Leclere, M., Neefs, E., Van Ransbeek, E., Berkenbosch, S., Clairquin, R., Muller, C., Forget, F., Hourdin, F., Talagrand, O., Rodin, A., Fedorova, A., Stepanov, A., Vinogradov, I., Kiselev, A., Kalinnikov, Yu., Durry, G., Sandel, B., and Stern, A., SPICAV: a Suite of Three Spectrometers to Study the Global Structure and Composition of the Venus Atmosphere, ESA SP1295, The Netherlands, Noordwijk, 2008 (in press).

8. Connes, P., Noxon, J.F., Traub, W.A., and Carleton, N., $\mathrm{O}_{2}\left({ }^{1} \Delta_{\mathrm{g}}\right)$ Emission in the Day and Night Airglow of Venus, Astrophys. J. Lett., 1979, vol. 233, pp. L29-L32.

9. Crisp, D. et al., Ground-Based Near-Infrared Observations of the Venus Nightside: $1.27-\mu \mathrm{m} \mathrm{O}_{2}\left({ }^{1} \Delta_{\mathrm{g}}\right)$ Airglow from the Upper Atmosphere, J. Geophys. Res., 1996, vol. 101, pp. 4577-4594.

10. Donahue, T. and Russell, C.T., The Venus Atmosphere and Ionosphere and Their Interaction with Solar Wind: an Overview (in Venus-2 book), 1997, pp. 3-32.

11. Drossart, P., Piccioni, G., Adriani, A., Angrilli, F., Arnold, G., Baines, K.H., Bellucci, G., Benkhoff, J., Bezard, B., Bibring, J.-P., Blanco, A., Blecka, M.I., Carlson, R.W., Coradini, A., Di Lellis, A., Encrenaz, T., Erard, S., Fonti, S., Formisano, V., Fouchet, T., Garcia, R., Haus, R., Helbert, J., Ignatiev, N.I., Irwin, P.G.J., Langevin, Y., Lebonnois, S., Lopez-Valverde, M.A., Luz, D., Marinangeli, L., Orofino, V., Rodin, A.V., RoosSerote, M.C., Saggin, B., Sanchez-Lavega, A., Stam, D.M., Taylor, F.W., Titov, D., Visconti, G., Zambelli, M., Hueso, R., Tsang, C.C.C., Wilson, C.F., and Afanasenko, T.Z., Scientific Goals for the Observation of Venus by VIRTIS on ESA/Venus Express Mission, Planet. Space Sci., 2007a, vol. 55, pp. 1653-1672.

12. Drossart, P., Piccioni, G., Gerard, J.C., Lopez-Valverde, M.A., Sanchez-Lavega, A., Zasova, L., Hueso, R., Taylor, F., Bezard, B., Adriani, A., Angrilli, F., Arnold, G., Baines, K.H., Bellucci, G., Benkhoff, J., Bibring, J.P., Blanco, A., Blecka, M.I., Carlson, R.W., Coradini, A., Di Lellis, A., Encrenaz, T., Erard, S., Fonti, S., Formisano, V., Fouchet, T., Garcia, R., Haus, R., Helbert, J., Ignatiev, N.I., Irwin, P., Langevin, Y., Lebonnois, S., Luz, D., Marinangeli, L., Orofino, V., Rodin, A.V., Roos-Serote, M.C., Saggin, B., Stam, D.M., Titov, D., Visconti, G., Zambelli, M., and Tsang, C., A Dynamic Upper Atmosphere of Venus as Revealed by VIRTIS in Venus Express, Nature, 2007b, vol. 450, pp. 641-645, doi:10.1038/nature06140.

13. Feldman, P.D., Moos, H.W., Clarke, J.T., and Lane, A.L., Identification of the UV Nightglow from Venus, Nature, 1979, vol. 279, pp. 221-222.

14. Formisano, V., Angrilli, F., Arnold, G., Atreya, S., Baines, K.H., Bellucci1, G., Bezard, B., Billebaud, F., Biondi, D., Blecka, M.I., Colangelil, L., Comolli1, L., Crisp, D., D’Amore, M., Encrenaz, T., Ekonomov, A., Esposito, F., Fiorenza, C., Fonti, S., Giuranna, M., Grassi, D., Grieger, B., Grigoriev, A., Helbert, J., Hirsch, H., Ignatiev, N., Jurewicz, A., Khatuntsev, I., Lebonnois, S., Lellouch, E., Mattana, A., Maturilli, A., Mencarelli, E., Michalska, M., Lopez-Moreno, J., Moshkin, B., Nespoli, F., Nikolsky, Yu., Nuccilli, F., Orleanski, P., Palomba, E., Piccioni, G., Rataj, M., Rinaldi, G., Rossi, M., Saggin, B., Stam, D., Titov, D., Visconti, G., and Zasova, L., The Planetary Fourier Spectrometer (PFS) Onboard the European Venus 
Express Mission, Planet. Space Sci., 2006, vol. 54, pp. 1298-1314.

15. Formisano, V., Angrilli, F., Arnold, G., Atreya, S., Baines, K.H., Bellucci, G., Bezard, B., Billebaud, F., Biondi, D., Blecka, M.I., Colangeli, L., Comolli, L., Crisp, D., D’Amore, M., Encrenaz, T., Ekonomov, A., Esposito, F., Fiorenza, C., Fonti, S., Giuranna, M., Grassi, D., Grieger, B., Helbert, J., Hirsch, H., Ignatiev, N., Jurewicz, A., Khatuntsev, I., Lebonnois, S., Lellouch, E., Mattana, A., Maturilli, A., Mencarelli, E., Michalska, M., Lopez Moreno, J., Moshkin, B., Nespoli, F., Nuccilli, F., Orleanski, P., Palomba, E., Piccioni, G., Rataj, M., Rinaldi, G., Rossi, M., Saggin, B., Stam, D., Titov, D., Visconti, G., and Zasova, L., The Planetary Fourier Spectrometer (PFS) Onboard the European Venus Express Mission, ESA SP-1295, The Netherlands, Noordwijk, 2008 (in press).

16. Fox, J.L., Advances in the Aeronomy of Venus and Mars, Adv. Space Res., 2004, vol. 33, pp. 132-139.

17. Gerard J.-C., Saglam, A., Piccioni, G., Drossart, P., Cox, C., Erard, S., Hueso, R., and Sanchez-Lavega, A., The Distribution of the $\mathrm{O}_{2}$ Infrared Nightglow Observed with VIRTIS on Board Venus Express, Geophys. Res. Lett., 2008, vol. 35, p. L02207, doi: 10.1029/2007GL032021.

18. Gierasch, P., Goody, R.M., Young, R.E., Crisp, D., Edwards, C., Kahn, R., McCleese, D., Rider, D., Del Genio, A., Greeley, R., Hou, A., Leovy, C.B., and Newman, M., The General Circulation of the Venus Atmosphere: an Assessment (in Venus-2 book), 1997, pp. 459-500.

19. Häusler, B., Pätzold, M., Tyler, G.L., Simpson, R.A., Bird, M.K., Dehant, V., Barriot, J.-P., Eidel, W., Mattei, R., Remus, S., Selle, J., Tellmann, S., and Imamura, T., Radio Science Investigations by VeRa Onboard the Venus Express Spacecraft, Planet. Space Sci., 2006, vol. 54, pp. 1315-1335.

20. Häusler, B., Pätzold, M., Tyler, G.L., Barriot, V.J.-P., Bird, M.K., Dehant, V., Hinson, D., Simpson, R.A., Treumann, R.A., Eidel, W., Mattei, R., Rosenblatt, P., Remus, S., Selle, J., and Tellmann, S., Venus Atmospheric, Ionospheric, Surface, and Interplanetary Radio Wave Propagation Studies with the Venus Express Radio Science Experiment VeRa, ESA SP-1295, The Netherlands, Noordwijk, 2008 (in press).

21. Lammer, H. et al., Loss of Water from Mars: Implications for the Oxidation of the Soil, Icarus, 2003, vol. 165, pp. 9-25.

22. Lellouch, E., Clancy, T., Crisp, D., Kliore, A.J., Titov, D., and Bougher, S.W., Monitoring of Mesospheric Structure and Dynamics (in Venus-2), Bougher, S.W., Hunten, D.M., and Phillips, R.J., Eds., University of Arizona Press, 1997, pp. 295-324.

23. Limaye, S.S., Venus Atmospheric Circulation: Known and Unknown, J. Geophys. Res., 2007, vol. 112, p. E04S09, doi:10.1029/206JE002814.

24. Limaye, S. S., Kossin, J. P., Rozoff, C., Piccioni, G., Titov, D. V., and Markiewicz, W. J., Vortex circulation on Venus: Dynamical similarities with terrestrial hurricanes, Geophys. Res. Lett., 2009, 36, L04204, doi:10.1029/2008GL036093.

25. Lopez-Valverde, M.A., Drossart, P., Carlson, R., Mehlman, R., and Roos-Serote, M., Non-LTE Infrared Obser- vations at Venus: From NIMS/Galileo to VIRTIS/Venus Express, Planet. Space Sci., 2007, vol. 55, pp. 17571771.

26. Markiewicz, W.J., Titov, D.V., Ignatiev, N., Keller, H.U., Crisp, D., Limaye, S.S., Jaumann, R., Moissl, R., Thomas, N., Esposito, L., Watanabe, S., Fiethe, B., Behnke, T., Szemerey, I., Michalik, H., Perplies, H., Wedemeier, M., Sebastian, I., Boogaerts, W., Dierker, C., Osterloh, B., Bker, W., Koch, M., Michaelis, H., Belyaev, D., Dannenberg, A., and Tschimmel, M., Venus Monitoring Camera for Venus Express, Planet. Space Sci., 2007a, vol. 55, pp. 1701-1711.

27. Markiewicz, W.J., Titov, D.V., Limaye, S.S., Keller, H.U., Ignatiev, N., Jaumann, R., Thomas, N., Michalik, H., Moissl, R., and Russo, P., Morphology and Dynamics of the Upper Cloud Layer of Venus, Nature, 2007b, vol. 450, pp. 633-636, doi:10.1038/nature06320.

28. Markiewicz, W.J., Titov, D.V., Fiethe, B., Behnke, T., Szemerey, I., Perplies, H., Wedemeier, M., Sebastian, I., Boogaerts, W., Dierker, C., Osterloh, B., Ignatiev, N., Beker, W., Koch, M., Keller, H.U., Jaumann, R., Michaelis, H., Michalis, H., Crisp, D., Esposito, L., Limaye, S.S., Watanabe, S., Thomas, N., Belyaev, D., Dannenberg, A., Tschimmel, M., and Moissl, R., Venus Monitoring Camera for Venus Express, ESA SP-1295, The Netherlands, Noordwijk, 2008 (in press).

29. McElroy, M.B., Prather, M.J., and Rodriguez, J.M., Loss of Oxygen from Venus, Geophys. Res. Lett., 1982, vol. 9, pp. 649-651.

30. Moissl, R., Khatuntsev, I., Limaye, S.S., Titov, D.V., Markiewicz, W.J., Ignatiev, N.I., Roatsch, T., Matz, K.-D., Jaumann, R., Almeida, M., Portyankina, G., Behnke, T., and Hviid, S.F., Venus cloud top winds from tracking UV features in Venus Monitoring Camera images, J. Geophys. Res., 2009, vol. 114, E00B31, doi: 10.1029/2008JE003117.

31. Moroz, V.I., Studies of the Atmosphere of Venus by Means of Spacecraft: Solved and Unsolved Problems, Adv. Space Res., 2002, vol. 29, pp. 215-225.

32. Müller, N., Helbert, J., Hashimoto, G. L., Tsang, C. C. C., Erard, S., Piccioni, G., and Drossart, P., Venus surface thermal emission at $1 " \mu \mathrm{m}$ in VIRTIS imaging observations: Evidence for variation of crust and mantle differentiation conditions, J. Geophys. Res., 2008, vol. 113, E00B17, doi: 10.1029/2008JE003118.

33. Müller, N., Herbert, J., Hashimoto, G., Tsang, C., Piccioni, G., and Drossart, P., The Surface of Venus in VIRTIS Imaging Observations, J. Geophys. Res., 2008 (in press).

34. Nakamura, M., Imamura, T., Ueno, M., Iwagami, N., Satoh, T., Watanabe, S., Taguchi, M., Takahashi, Y., Suzuki, M., Abe, T., Hashimoto, G.L., Sakanoi, T., Okano, S., Kasaba, Y., Yoshida, J., Yamada, M., Ishii, N., Yamada, Y., Uemizu, K., Fukuhara, T., and Oyama, K.I., Planet-C: Venus Climate Orbiter Mission of Japan, Planet. Space Sci., 2007, vol. 55, pp. 1831-1842.

35. Ohtsuki, S. et al., Ground-Based Observation of the Venus 1.27- $\mathrm{mm} \mathrm{O}_{2}$ Airglow, Adv. Space Res., 2005, vol. 36, pp. 2038-2042.

36. Pätzold, M., Häusler, B., Bird, M.K., Tellmann, S., Mattei, R., Asmar, S.W., Dehant, V., Eidel, W., Imamura, T., Simpson, R.A., and Tyler, G.L., The Structure of Venus' Middle Atmosphere and Ionosphere, Nature, 2007, vol. 450, pp. 657-660. 
37. Piccialli, A., Titov, D.V., Grassi, D., et al., Cyclostrophic winds from the Visible and Infrared Thermal Imaging Spectrometer temperature sounding: A preliminary analysis, J. Geophys. Res., 2008, vol. 113, E00B11, doi: 10.1029/2008JE003127.

38. Piccioni, G., Drossart, P., Sanchez-Lavega, A., Hueso, R., Taylor, F.W., Wilson, C.F., Grassi, D., Zasova, L., Moriconi, M., Adriani, A., Lebonnois, S., Coradini, A., Bezard, B., Angrilli, F., Arnold, G., Baines, K.H., Bellucci, G., Benkhoff, J., Bibring, J.-P., Blanco, A., Blecka, M.I., Carlson, R.W., Di Lellis, A., Encrenaz, T., Erard, S., Fonti, S., Formisano, V., Fouchet, T., Garcia, R., Haus, R., Helbert, J., Ignatiev, N.I., Irwin, P.G.J., Langevin, Y., Lopez-Valverde, M.A., Luz, D., Marinangeli, L., Orofino, V., Rodin, A.V., Roos-Serote, M.C., Saggin, B., Stam, D.M., Titov, D., Visconti, G., and Zambelli, M., The VIRTIS-Venus Express Technical Team, South-Polar Features on Venus Similar to Those Near the North Pole, Nature, 2007, vol. 450, pp. 637640, doi: 10.1038/nature06209.

39. Piccioni, G., Drossart, P., Suetta, E., Cosi, M., Ammannito, E., Barbis, A., Berlin, R., Boccaccini, A., Bonello, G., Bouye, M., Capaccioni, F., Cherubini, G., Dami, M., Dupuis, O., Fave, A., Filacchione, G., Hello, Y., Henry, F., Hofer, S., Huntzinger, G., Melchiorri, R., Parisot, J., Pasqui, C., Peter, G., Pompei, C., Reess, J.M., Semery, A., Soufflot, A., Adriani, A., Angrilli, F., Arnold, G., Baines, K., Bellucci, G., Benkhoff, J., Bezard, B., Bibring, J.-P., Blanco, A., Blecka, M.I., Carlson, R., Coradini, A., Di Lellis, A., Encrenaz, T., Erard, S., Fonti, S., Formisano, V., Fouchet, T., Garcia, R., Haus, R., Helbert, J., Ignatiev, N.I., Irwin, P., Langevin, Y., Lebonnois, S., Lopez Valverde, M.A., Luz, D., Marinangeli, L., Orofino, V., Rodin, A., Roos-Serote, M.C., Saggin, B., Sanchez-Lavega, A., Stam, D., Taylor, F., Titov, D.V., Visconti, G., and Zambelli, M., VIRTIS (Visible and Infrared Thermal Imaging Spectrometer) for Venus Express, ESA SP-1295, The Netherlands, Noordwijk, 2008 (in press).

40. Russell, C.T., Zhang, T.L., Delva, M., Magnes, W., Strangeway, R.J., and Wei1, H.Y., Lightning on Venus Inferred from Whistler-Mode Waves in the Ionosphere, Nature, 2007, vol. 450, pp. 661-662.

41. Sanchez-Lavega, A., Hueso, R., Piccioni, G., Drossart, P., Peralta, J., Perez-Hoyos, S., Wilson, C.F., Taylor, F.W., Baines, K.H., Luz, D., Erard, S., and Lebonnois, S., Variable Winds on Venus Mapped in Three Dimensions, Geophys. Res. Lett., 35, L13204, doi: 10.1029/2008GL033817, 2008.

42. Schubert, G., General Circulation and the Dynamical State of the Venus Atmosphere, in Venus, Hunten, D.M., Colin, L., Donahue, T.M., and Moroz, V.I., Eds., Ticson, Univ. of Arizona Press, 1983, pp. 681-765.

43. Schubert, G., Bougher, S.W., Covey, C.C., Del Genio, A.D., Grossman, A.S., Hollingsworth, J.L., Limaye, S.S., and Young, R.E., Venus Atmosphere Dynamics: a Continuing Enigma, in AGU Geophysical Monograph 176 Exploring Venus as Terrestrial Planet, Esposito, L.W., Stofan, E.R., and Cravens, Th.E., Eds., 2007, pp. 121-138.

44. Sieff, A., Schofield, J.T., Kliore, A.J., Taylor, F.W., Limaye, S.S., Revercomb, H.E., Sromovsky, L.A.,
Kerzhanovich, V.V., Moroz, V.I., and Marov, M.Ya., Models of the Structure of the Atmosphere of Venus from the Surface to 100 Kilometers Altitude, Adv. Space. Res., 1985, vol. 5, no. 1, pp. 3-58.

45. Stewart, A.I.F., Gerard, J.-C., Rusch, D.W., and Bougher, S.W., Morphology of the Venus Ultraviolet Night Airglow, J. Geophys. Res., 1980, vol. 85, pp. 7861-7870.

46. Svedhem, H., Titov, D.V., McCoy, D., Lebreton, J.-P., Barabash, S., Bertaux, J.-L., Drossart, P., Formisano, V., Häusler, B., Korablev, O., Markiewicz, W.J., Nevejans, D., Pätzold, M., Piccioni, G., Zhang, T.L., Taylor, F.W., Lellouch, E., Koschny, D., Witasse, O., Warhaut, M., Accommazzo, A., Rodriguez-Canabal, J., Fabrega, J., Schirman, T., Clochet, A., and Coradini, M., Venus Express-the First European Mission to Venus, Planet. Space Sci., 2007a, vol. 55, pp. 1636-1652.

47. Svedhem, H., Titov, D.V., Taylor, F.W., and Witasse, O., Venus as a More Earth-Like Planet, Nature, 2007b, vol. 450, pp. 629-632.

48. Svedhem, H., McCoy, D., Titov, D.V., Taylor, F.W., Witasse, O., Venus Express-Science Objectives and Mission Overview, ESA SP-1295, The Netherlands, Noordwijk, 2008 (in press)

49. Taylor, F.W., Venus Before Venus Express, Planet. Space Sci., 2006, vol. 54, pp. 1249-1262.

50. Taylor, F.W., et al., Structure and meteorology of the middle atmosphere of Venus: infrared remote sensing from the Pioneer orbiter, J. Geophys. Res., 1980, vol. 85, pp. 7963-8006.

51. Titov, D.V., Lellouch, F.W., Marinangeli, L., and Opgenoorth, H., The Venus Express Team. Venus Express: an Orbiter for the Study of the Atmosphere, the Plasma Environment, and the Surface of Venus, Mission Definition Report, ESA-SCI, 2001, p. 6.

52. Titov, D.V., Svedhem, H., and Taylor, F.W., The Atmosphere of Venus: Current Knowledge and Future Investigations, in Solar System Update, Blondel, $\mathrm{Ph}$. And Mason, J.W., Eds., Springer-Praxis, 2006a, pp. 87-110.

53. Titov, D.V., Svedhem, H., McCoy, D., Lebreton, J.-P., Barabash, S., Bertaux, J.-L., Drossart, P., Formisano, V., Häusler, B., Korablev, O.I., Markiewicz, W.J., Nevejans, D., Pätzold, M., Piccioni, G., Zhang, T.L., Taylor, F.W., Lellouch, E., Koschny, D., Witasse, O., Warhaut, M., Accomazzo, A., Rodriguez-Canabal, J., Fabrega, J., Schirmann, T., Clochet, A., and Coradini, M., Venus Express: Scientific Goals, Instrumentation and Scenario of the Mission, Cosmic Research, 2008b, vol. 44, no. 4, pp. 334-348.

54. Titov, D., Svedhem, H., Koschny, D., Hoofs, R., Barabash, S., Bertaux, J.-L., Drossart, P., Formisano, V., Häusler, B., Korablev, O., Markiewicz, W.J., Nevejans, D., Pätzold, M., Piccioni, G., Zhang, T.L., Merritt, D., Witasse, O., Zender, J., Accommazzo, A., Sweeney, M., Trillard, D., Janvier, M., and Clochet, A., Venus Express Science Planning, Planet. Space Sci., 2006c, vol. 54, nos. 13-14, pp. 1279-1297.

55. Titov, D.V., Bullock, M., Crisp, D., Renno, N., Taylor, F.W., and Zasova, L.V., Radiation in the Atmosphere of Venus, in AGU Geophysical Monograph 176 "Exploring Venus as Terrestrial Planet”, Esposito, L.W., Stofan, E.R., and Cravens, Th.E., Eds., 2007, pp. 121-138. 
56. Titov, D.V., Taylor, F.W., Svedhem, H., et al., Atmospheric structure and dynamics as the cause of ultraviolet markings in the clouds of Venus, Nature, 2008, vol. 456, pp. 620-623.

57. Zasova, L.V., Ignatiev, N.I., Khatuntsev, I., Linkin, V.M., Structure of the Venus atmosphere, Planet. Space Sci., 2007, vol. 55, pp. 1712-1728.

58. Zhang, T.L., Baumjohann, W., Delva, M., Auster, H.-U., Balogh, A., Russell, C.T., Barabash, S., Balikhin, M., Berghofer, G., Biernat, H.K., Lammer, H., Lichtenegger, H., Magnes, W., Nakamura, R., Penz, T., Schwingenschuh, K., Voros, Z., Zambelli, W., Fornacon, K.-H., Glassmeier, K.-H., Richter, I., Carr, C., Kudela, K., Shi, J.K., Zhao, H., Motschmann, U., and Lebreton, J.-P., Magnetic Field Investigation of the Venus Plasma Environment: Expected New Results from Venus Express, Planet. Space Sci., 2006, vol. 54, pp. 13361343.
59. Zhang, T.L., Berghofer, G., Magnes, W., Delva, M., Baumjohann, W., Biernat, H., Lichtenegger, H., Nakamura, R., Schwingenschuh, K., Auster, H.-U., Fornacon, K.-H., Richter, I., Glassmeier, K.-H., Carr, C., Balogh, A., Barabash, S., Kudela, K., Balikhin, M., Russell, C.T., Motschmann, U., and Lebreton, J.-P., The Fluxgate Magnetometer for the Venus Express Mission, ESA SP-1295, The Netherlands, Noordwijk, 2008 (in press).

60. Zhang, T.L., Delva, M., Baumjohann, W., Auster, H.-U., Carr, C., Russell, C.T., Barabash, S., Balikhin, M., Kudela, K., Berghofer, G., Biernat, H.K., Lammer, H., Lichtenegger, H., Magnes, W., Nakamura, R., Schwingenschuh, K., Volwerk, M., Voeroes, Z., Zambelli, W., Fornacon, K.-H., Glassmeier, K.-H., Richter, I., Balogh, A., Schwarzl, H., Pope, S.A., Shi, J.K., Wang, C., Motschmann, U., and Lebreton, J.P., Little or No Solar Wind Enters Venus' Atmosphere at Solar Minimum, Nature, 2007, vol. 450, pp. 654-656. 